Math. Model. Nat. Phenom.

Vol. 6, No. 4, 2011, pp. 118-150

DOI: $10.1051 / \mathrm{mmnp} / 20116406$

\title{
Homogeneous Cooling with Repulsive and Attractive Long-Range Potentials
}

\author{
M.K. Müller and S. Luding * \\ Multi Scale Mechanics, CTW, UTwente, P. O. Box 217, 7500 AE Enschede, Netherlands
}

\begin{abstract}
The interplay between dissipation and long-range repulsive/attractive forces in homogeneous, dilute, mono-disperse particle systems is studied. The pseudo-Liouville operator formalism, originally introduced for hard-sphere interactions, is modified such that it provides very good predictions for systems with weak long-range forces at low densities, with the ratio of potential to fluctuation kinetic energy as control parameter. By numerical simulations, the theoretical results are generalized with empirical, density dependent correction-functions up to moderate densities.

The main result of this study on dissipative cooling is an analytical prediction for the reduced cooling rate due to repulsive forces and for the increased rate due to attractive forces. In the latter case, surprisingly, for intermediate densities, similar cooling behavior is observed as in systems without long-range interactions. In the attractive case, in general, dissipation leads to inhomogeneities earlier and faster than in the repulsive case.
\end{abstract}

Key words: granular gas, hydrodynamics, long-range forces, numerical simulations AMS subject classification: 76T25, 76P05, 35Q35

\section{Introduction}

Granular materials are assemblies of meso- and macroscopic particles and differ from molecular systems mainly due to the size of the particles $[33,4,19]$ and the type of interactions. Their theoretical description uses the laws of classical mechanics, while molecular interactions are successfully approached by, e.g., density functionals and quantum-theory. In contrast to molecules, macroscopic particles typically interact inelastically, i.e., kinetic energy is converted into other forms of energy such as potential energy (reversible deformation), irreversible (plastic) deformation, vibrations, sound and heat. The large number of internal degrees of freedom of macroscopic

\footnotetext{
*Corresponding author E-mail: s.luding@utwente.nl
} 
particles makes it practically impossible to retrieve kinetic energy dissipated during collisions.

Granular gases [72] are granular materials where the duration of a particle-particle collision is much shorter than the time between two collisions. This situation can be procured by either placing a dilute particle system in a gravity free (or micro-gravitational) environment $[6,43,5]$ or - financially much cheaper - by feeding the system with energy from outside such that a granular gas in steady state forms (e.g., by vertically vibrating the enclosure, see [24, 70] and references therein). Another, naturally occurring example for a granular gas is represented by the rings orbiting the outer planets of the Solar System [8, 30, 9, 26, 31, 62, 78] or even by the large-scale distribution of galaxies [79].

In a granular gas in the dilute limit, one will detect mainly binary instead of multiple particle collisions. The assumption of binary collisions in a many-body system is important for the theoretical description, see Section 2.. The persistent loss of kinetic energy due to contacts not only makes the gas cool down but is also accompanied by collective phenomena: granular gases are subject to instabilities and cluster formation $[57,29,58,70,54,50,59,60]$, deviations from the Maxwell-Boltzmann velocity distribution with both constant and velocity-dependent dissipation $[13,77,10,11,37,36]$, phase transitions $[25,70]$ and the formation of vortices $[71,48]$. In brief, granular media can exhibit fluidic phenomena [46] and even have different "states of aggregation" in coexistence $[25,24]$.

The hydrodynamic instability that leads to cluster formation is exclusively an effect of dissipation during collisions [57, 59,60], and thus should be enhanced by attractive potentials and diminished by repulsive potentials between the particles. Electrically equally charged granular media with repulsive long-range interactions are in nature and industrial processes rather the rule than an exception, e.g., granular pipe flow [85], suspensions and fluids with charged particles $[82,84,16,17,45,40]$, electro-sorting in waste disposal processing [69], electrophotography in print processes [15, 39] and electrospraying of pesticides in greenhouses [2, 28, 66]. Electrostatically charged macroscopic solid particles can be obtained by procuring repeated mechanical contacts between them under dry conditions ("triboelectrification", see [47, 68]), but this problem is beyond the scope of this article. On the other hand, particle systems with attractive long-range interactions [18] can be found in both electrostatic coating processes $[42,83]$ and in space, where huge mass distributions such as dense granular rings and disks around central bodies are affected by very strong self-gravitation ([63] and references therein). This article, however, is devoted to the collisional cooling behavior of granular media with either (long-range) repulsive or attractive potentials in the dilute limit.

For better understanding the pseudo-Liouville formalism, in Chapter 2. we will explain the basics of the two-particle mechanics, including long-range repulsive and attractive interaction forces. (For more advanced theories, see Refs. [21, 23].) Thereafter, we will introduce to the many-particle mechanics as described by the pseudo-Liouville operator formalism. Then, we will extend this theory for long-range interactions, while considering the dissipative nature of the particles. We will provide the mathematical details in Appendix A and discuss the corrections in Appendix B.

In Chapter 3., we discuss our "experimental" set-up we use to check the new theory. (Since numerical results from Direct Simulation Monte Carlo (DSMC) were shown to agree nicely with Molecular Dynamics (MD) particle simulations in the low density cases up to moderate density and 
dissipation [61], we do not present DSMC results here.) In the final Chapter 4., we will present the results of our study. Classical systems without long-range forces represent a special case of the theory. The extended pseudo-Liouville operator formalism works well for weak long-range interaction forces, weak dissipation and low densities because it does not consider the influence of multi-particles effects. We then expand our study towards higher densities and thus extend the theory correspondingly - but this time on an empirical basis.

\section{Theory}

Realistic many-body systems involve arbitrarily complex interaction potentials between their macroscopic constituents. The hard sphere assumption is a simplified model, where the repulsive contact potential is infinitely strong (excluded volume) and the contact duration is infinitely short. As a consequence, multiple particle contacts cannot occur. In the following, a theoretical tool, the pseudo-Liouville operator formalism, will be further developed to include the effect of long-range interactions. Then it can be used to describe the dynamics of a many-body system as a sequence of binary collisions [65].

As long as the system conserves total energy (elastic collisions), the time derivative of any time-dependent observable can be expressed by the Hamilton operator that contains spatial derivatives (see $[34,11]$ and textbooks on classical mechanics). The application of spatial derivatives to the spatially discontinuous contact potential of hard spheres causes analytical problems. Therefore, in order to preserve the formal structure of the Liouville equation, only those particle pairs are considered that fulfill specific "collision rules", which are discussed below. As a theoretical tool that meets these requirements, the pseudo-Liouville operator was introduced [22] and successfully applied to various situations $[41,55,81]$. However, contrary to the premises mentioned above, this study will show that the pseudo-Liouville operator theory can be adapted to a dissipative hard sphere gas with smooth, long-range interaction potentials - at least in the dilute limit.

\subsection{Two-particle mechanics}

Without loss of generality, we discuss spherical particles $j$ and $k$ of identical, uniform mass $m$ and radius $a$. The long-range particle pair interaction is described by a repulsive or attractive potential

$$
\Phi\left(r_{j k}\right)=-\frac{\alpha}{r^{s}}
$$

where $r=\left|\boldsymbol{r}_{j k}\right|=\left|\boldsymbol{r}_{j}-\boldsymbol{r}_{k}\right|$ is the absolute value of the relative distance vector between the particle centers. $\alpha$ contains all parameters of the interaction potential, where $\alpha<0$ denotes repulsive and $\alpha>0$ attractive interactions. Since we deal with conservative long-range interaction potentials, the force on particle $j$ exerted by $k$ can be obtained from Eq. (2.1) via

$$
\boldsymbol{F}_{j}^{\text {long }}=-\nabla_{r} \Phi(r)=-\frac{s \alpha}{r^{s+1}} \hat{\boldsymbol{r}}
$$


(a)

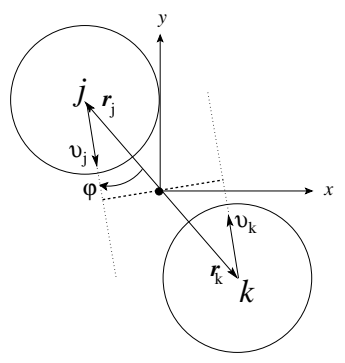

(b)

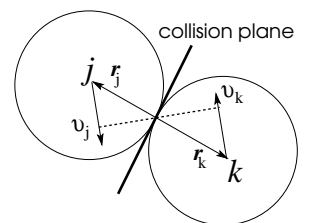

(c)

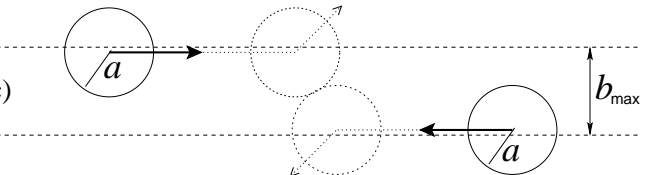

(d)

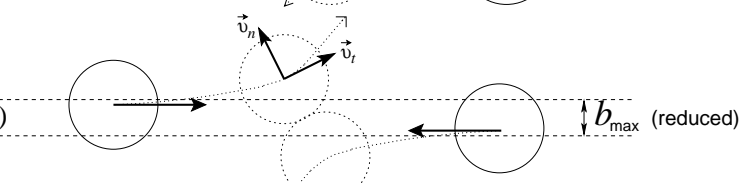

(e)

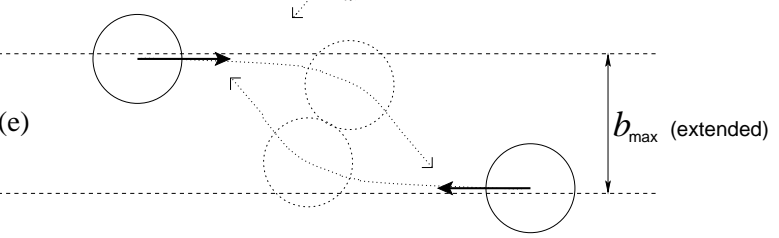

Figure 1: Schematic representation of collisions. A typical encounter of two arbitrary particles $j$ and $k$ in the center-of-mass system, where the coordinate system is located in the center of mass, before contact (a) and at contact (b), where the length of the dotted line indicates the actual impact parameter, $b$. In the force-free case (c) the maximum impact parameter $b_{\max }$ equals the sum of the radii as indicated by the dashed line; in the repulsive case (d) it is reduced, whereas in the attractive case (e) it is extended, see Eq. (2.7), as compared to case (c).

where $\hat{\boldsymbol{r}}=\left(\boldsymbol{r}_{j}-\boldsymbol{r}_{k}\right) /\left|\boldsymbol{r}_{j}-\boldsymbol{r}_{k}\right|$ points from $k$ to $j$ and the hat denotes the unit vector. Note that $\boldsymbol{F}_{k}^{\text {long }}=-\boldsymbol{F}_{j}^{\text {long }}$ because of the action=reaction principle. The power of the potential is $s \geq 1$, where $s=1$ corresponds to the longest possible range of a potential. This "worst case" will be considered in the results section 4 .. In order to describe the two-particle encounter, we consider the coordinate system in the center of mass of the binary system, see Fig. 1, with the particle positions $\boldsymbol{r}_{j}=-\boldsymbol{r}_{k}$ and velocities $\boldsymbol{v}_{j}=-\boldsymbol{v}_{k}$ in the $x$ - $y$-plane. Introducing abbreviations for the relative velocity vector, $\boldsymbol{v}=\boldsymbol{v}_{j}-\boldsymbol{v}_{k}=2 \boldsymbol{v}_{j}=-2 \boldsymbol{v}_{k}$, the relative distance vector, $\boldsymbol{r}=\boldsymbol{r}_{j}-\boldsymbol{r}_{k}=2 \boldsymbol{r}_{j}=$ $-2 \boldsymbol{r}_{k}$ and $\varphi=\operatorname{acos}(\hat{\boldsymbol{v}} \cdot \hat{\boldsymbol{r}})$, we can obtain the impact parameter, $b$, from $\boldsymbol{v} \times \boldsymbol{r}=v r \sin (\varphi) \hat{\boldsymbol{s}}=v b \hat{\boldsymbol{s}}$ with $\hat{s}$ pointing out of the plane of view. The total energy of the binary system is

$$
E=\frac{1}{2} m\left(\boldsymbol{v}_{j}^{2}+\boldsymbol{v}_{k}^{2}\right)+\Phi(r)=\frac{1}{4} m \boldsymbol{v}^{2}+\Phi(r)=\frac{1}{4} m\left(v_{n}^{2}+v_{t}^{2}\right)+\Phi(r),
$$

where $v_{n}=\boldsymbol{v} \cdot \hat{\boldsymbol{r}}$ and $v_{t}=\boldsymbol{v} \cdot \hat{\boldsymbol{t}}$ are the components of the relative velocity, $\boldsymbol{v}$, normal and tangential (parallel) to the collision plane, see Fig. 1, i.e., $\boldsymbol{v}=v_{n} \hat{\boldsymbol{r}}+v_{t} \hat{\boldsymbol{t}}$. Note, that we define the normal relative velocity of both particles as to be negative if they approach each other and to be positive if they separate from each other. The total angular momentum of the binary system [51] is

$$
\boldsymbol{L}=m\left(\boldsymbol{r}_{j} \times \boldsymbol{v}_{j}\right)+m\left(\boldsymbol{r}_{k} \times \boldsymbol{v}_{k}\right)=\frac{1}{2} m(\boldsymbol{r} \times \boldsymbol{v})=\frac{1}{2} m b v \hat{\boldsymbol{s}}=\frac{1}{2} m b \sqrt{v_{n}^{2}+v_{t}^{2}} \hat{\boldsymbol{s}} .
$$

We consider three cases:

(i) For two approaching particles, which are "infinitely" far apart from each other (subscript " $\infty$ "), Eq. (2.3) simplifies to $E_{\infty}=(1 / 4) m\left(\boldsymbol{v}_{\infty} \cdot \hat{\boldsymbol{r}}\right)^{2}$, since $\boldsymbol{v}_{\infty} \cdot \hat{\boldsymbol{t}} \approx 0$ and $\Phi\left(r_{\infty}\right) \approx 0$. Eq. (2.4) then 
leads to the angular momentum at infinity $\boldsymbol{L}_{\infty}=(1 / 2) m b_{\infty}\left(\boldsymbol{v}_{\infty} \cdot \hat{\boldsymbol{r}}\right) \hat{\boldsymbol{s}}$.

(ii) When both particles are at closest distance without contact during their fly-by (subscript " 0 "), Eq. (2.3) becomes $E_{0}=(1 / 4) m\left(\boldsymbol{v}_{0} \cdot \hat{\boldsymbol{t}}\right)^{2}+\Phi\left(r_{0}\right)$, where $\boldsymbol{v}_{0} \cdot \hat{\boldsymbol{r}}=0$ and $b_{0}=r_{0}$, so that Eq. (2.4) yields $\boldsymbol{L}_{0}=(1 / 2) m r_{0}\left(\boldsymbol{v}_{0} \cdot \hat{\boldsymbol{t}}\right) \hat{\boldsymbol{s}}$.

(iii) The contact situation is denoted by the subscript "c" and leads to $E_{c}=(1 / 4) m\left[\left(\boldsymbol{v}_{c} \cdot \hat{\boldsymbol{r}}\right)^{2}+\right.$ $\left.\left(\boldsymbol{v}_{c} \cdot \hat{\boldsymbol{t}}\right)^{2}\right]+\Phi\left(r_{c}\right)$ and $\boldsymbol{L}_{c}=(1 / 2) m b_{c}\left[\left(\boldsymbol{v}_{c} \cdot \hat{\boldsymbol{r}}\right)^{2}+\left(\boldsymbol{v}_{c} \cdot \hat{\boldsymbol{t}}\right)^{2}\right]^{1 / 2} \hat{\boldsymbol{s}}$. For the conservation of total energy follows

$$
\frac{1}{4} m\left(\boldsymbol{v}_{\infty} \cdot \hat{\boldsymbol{r}}\right)^{2}=\frac{1}{4} m\left(\boldsymbol{v}_{0} \cdot \hat{\boldsymbol{t}}\right)^{2}+\Phi\left(r_{0}\right)=\frac{1}{4} m\left[\left(\boldsymbol{v}_{c} \cdot \hat{\boldsymbol{r}}\right)^{2}+\left(\boldsymbol{v}_{c} \cdot \hat{\boldsymbol{t}}\right)^{2}\right]+\Phi\left(r_{c}\right)
$$

and for the conservation of total angular momentum follows

$$
\frac{1}{2} m b_{\infty}\left(\boldsymbol{v}_{\infty} \cdot \hat{\boldsymbol{r}}\right) \hat{\boldsymbol{s}}=\frac{1}{2} m r_{0}\left(\boldsymbol{v}_{0} \cdot \hat{\boldsymbol{t}}\right) \hat{\boldsymbol{s}}=\frac{1}{2} m b_{c} \sqrt{\left(\boldsymbol{v}_{c} \cdot \hat{\boldsymbol{r}}\right)^{2}+\left(\boldsymbol{v}_{c} \cdot \hat{\boldsymbol{t}}\right)^{2}} \hat{\boldsymbol{s}}
$$

From Eq. (2.6), one can obtain for the tangential relative velocity at the closest distance, $\boldsymbol{v}_{0} \cdot \hat{\boldsymbol{t}}=$ $b_{\infty}\left(\boldsymbol{v}_{\infty} \cdot \hat{\boldsymbol{r}}\right) / r_{0}$. Plugging this into Eq. (2.5), allows to solve for the initial impact parameter

$$
b_{\infty}\left(r_{0}\right)=r_{0}\left(1-\frac{4 \Phi\left(r_{0}\right)}{m\left(\boldsymbol{v}_{\infty} \cdot \hat{\boldsymbol{r}}\right)^{2}}\right)^{1 / 2} .
$$

If we define the closest distance as the contact distance, $r_{0}=r_{c}=2 a, b_{\max }:=b_{\infty}(2 a)$ is the maximum impact parameter: The actual initial impact parameter has to be smaller than $b_{\max }$ in order to have a collision. In absence of long-range forces, i.e., $\alpha=0$, the maximum impact parameter equals $2 a$, see case (c) in Fig. 1. For the repulsive case, $\alpha<0, \Phi>0$, the maximum collision parameter is reduced (d), whereas for the attractive case, $\alpha>0, \Phi<0$, it is enlarged (e), relative to the case $\alpha=0$.

Long-range forces are permanently acting on the particles via Eq. (2.2) and can decrease $(\alpha<$ $0)$ or increase $(\alpha>0)$ the probability of dissipative collisions. Now, let us assume a head-on collision of the particles, i.e., $b_{\infty}(2 a)=0$, which makes the total angular momentum, Eq. (2.6), vanish. We now compute from Eq. (2.5) velocities that correspond to the lowest total energy that allows for a collision and a complete separation of the particles as well. In the repulsive case, this corresponds to zero contact velocity and in the attractive case this corresponds to zero velocity at infinity, so that the potential at contact determines the critical velocity:

$$
\Phi(2 a)= \pm \frac{1}{4} m\left(\boldsymbol{v}_{\mathrm{cr}} \cdot \hat{\boldsymbol{r}}\right)^{2}= \begin{cases}+\frac{1}{4} m\left(\boldsymbol{v}_{n, b} \cdot \hat{\boldsymbol{r}}\right)^{2}>0 & \text { for repulsion } \\ -\frac{1}{4} m\left(\boldsymbol{v}_{n, e} \cdot \hat{\boldsymbol{r}}\right)^{2}<0 & \text { for attraction }\end{cases}
$$

In case of repulsion, $\Phi(2 a)>0$, the contact potential energy is positive and two approaching particles far from each other have to overcome the critical velocity in order to collide. In case of attraction, $\Phi(2 a)<0$, the contact potential and the kinetic energy are negative and two separating particles at contact must overcome the critical velocity in order to escape from each other to infinity. For lower total energy, no collision takes place (due to repulsion), or the particles do not separate 
and remain in their neighborhood (due to attraction). In the repulsive case, we define $v_{\mathrm{cr}}:=v_{n, b}$ and the corresponding contact potential, $\Phi(2 a)$, we refer to as the repulsive energy $(b)$ arrier. In the attractive case, we define $v_{\mathrm{cr}}:=v_{n, e}$ and the contact potential is referred to as the attractive (e)scape potential.

The second ingredient of our model are the contacts as discussed in the following subsection.

\subsection{Collision mechanics}

In the following, we focus on a collision between the particles $j$ and $k$. Keeping this in mind, we will drop the index "c" for convenience. If hard sphere particles collide, see Fig. 1 (b) with $r=2 a$ and $b_{\infty} \neq 0$, they will change their translational relative momentum. Their relative velocity just before the collision (unprimed) will be instantaneously replaced by the one just after (primed):

$$
\boldsymbol{v} \rightarrow \boldsymbol{v}^{\prime}=\boldsymbol{v}+\frac{2 \Delta \boldsymbol{p}}{m}
$$

where the change of relative momentum in normal direction, $(\Delta \boldsymbol{p} \cdot \hat{\boldsymbol{r}}) \hat{\boldsymbol{r}}= \pm(1 / 2) m(1+e) v_{n} \hat{\boldsymbol{r}}$, is accompanied by a loss of kinetic energy. The coefficient of normal restitution, $e$, defined via $\boldsymbol{v}_{n}^{\prime} \cdot \hat{\boldsymbol{r}}^{\prime}=-e \boldsymbol{v}_{n} \cdot \hat{\boldsymbol{r}}$ - where for rigid spheres the normal direction does not change during contact $\hat{\boldsymbol{r}}^{\prime}=\hat{\boldsymbol{r}}$ - quantifies which fraction of the normal relative speed remains, i.e., $0<e<1$. This leads to a change of kinetic energy

$$
\Delta E_{\mathrm{kin}}=-\frac{1}{4} m\left(1-e^{2}\right) v_{n}^{2}
$$

that depends on the normal impact velocity just before the collision.

In our soft particle simulations, a physical contact leads to a strong repulsion force between both particles, which increases linearly with the overlap. The overlap is quantified by $(2 a-r)$ and is positive if the particles are in contact. The repulsive short-range force on particle $j$ is complemented by a dissipation term, according to the spring dashpot model, so that

$$
\boldsymbol{F}_{j}^{\text {short }}=\left[k(2 a-r)+\gamma_{n} v_{n}\right] \hat{\boldsymbol{r}}
$$

where $k$ defines the stiffness of the contact and $\gamma_{n}$ is a viscous damping coefficient that is of empirical origin and has to be chosen in our simulations such that the desired coefficient of normal restitution $e$ with dissipation $\left(1-e^{2}\right)$ is obtained. The overlap is an oscillating function of time $[49,51]$ with the damped frequency $\omega=\sqrt{(2 k / m)-\left(\gamma_{n} / m\right)^{2}}$ and the theoretical contact duration $t_{c}=\pi / \omega$. Note again, that $v_{n}<0$ for approaching particles (and the overlap increases) and $v_{n}>0$ for separating particles during contact (and the overlap decreases). Since $v_{n}$ is the time derivative of the overlap, we can express the coefficient of restitution in terms of the simulation parameters $k$ and $\gamma_{n}$ by using the relation $\boldsymbol{v}_{n}^{\prime} \cdot \hat{\boldsymbol{r}}^{\prime}=-e \boldsymbol{v}_{n} \cdot \hat{\boldsymbol{r}}$ - where the normal direction can change during contact - leading to $e=\exp \left(-t_{c} \gamma_{n} / m\right)$.

After having discussed the underlying models for the two-particle interaction, we will now turn to the statistical description of the cooling behavior of many particles. 


\subsection{Many-particle mechanics at low densities}

The pseudo-Liouville operator is a convenient tool to describe inelastic hard core collisions in many-body systems [22, 41, 55, 81]. Since we are interested in the cooling behavior of manyparticle systems with a long-range interaction potential on top of the usual hard core, we "correct" the existing results, but first introduce the classical theory. In the following calculations we will consider the total kinetic energy, $E_{\text {kin }}(t)$. The pseudo-Liouville operator, $\mathcal{L}^{+}$, is composed of a free streaming and an interaction part that only selects pairs of colliding particles $j$ and $k$. Applying $\mathcal{L}^{+}$to the kinetic energy, yields the change of kinetic energy per unit time:

$i \mathcal{L}^{+} E_{\text {kin }}(t):=\sum_{j=1}^{N} \boldsymbol{v}_{j} \frac{\partial}{\partial \boldsymbol{r}_{j}} E_{\text {kin }}(t)+\sum_{j=1}^{N-1} \sum_{k=j+1}^{N}\left|\boldsymbol{v}_{j k} \cdot \hat{\boldsymbol{r}}_{j k}\right| \Theta\left(-\boldsymbol{v}_{j k} \cdot \hat{\boldsymbol{r}}_{j k}\right) \delta\left(\left|\boldsymbol{r}_{j k}\right|-2 a\right)\left(b_{j k}^{+}-1\right) E_{\text {kin }}(t)$,

where $i$ is the imaginary unit and $N$ the particle number. $\Theta(\cdot)$ is the Heaviside step function, which is zero if its argument is negative and unity otherwise. $\delta(\cdot)$ denotes Kronecker's delta symbol, which is zero except for zero argument, and $b_{j k}^{+}$is the binary collision operator that instantaneously replaces the velocities of both particles just before the collision by the corresponding values just after, see Eq. (2.9) in subsection 2.2..

In the absence of volume forces and long-range interactions, the free streaming part (first term) will not change the kinetic energy. Thus, in the following, we first consider only the interaction part (second term), which fulfills the "collision rules" mentioned above: $\left|\boldsymbol{v}_{j k} \cdot \hat{\boldsymbol{r}}_{j k}\right|$ is the normal component of the relative velocity of the particle pair in question and means that faster particles can collide more often per unit time. $\Theta\left(-\boldsymbol{v}_{j k} \cdot \hat{\boldsymbol{r}}_{j k}\right)$ selects only those particle pairs whose relative normal velocities are directed such that they approach each other, i.e., when $\boldsymbol{v}_{j k} \cdot \hat{\boldsymbol{r}}_{j k}<0$. The angle $\theta$ between both vectors must be in the range $\pi / 2<\theta<3 \pi / 2$ for a collision to be possible. $\delta\left(\left|\boldsymbol{r}_{j k}\right|-2 a\right)$ makes sure that only pairs of hard spheres are selected that are in physical contact with each other. $\left(b_{j k}^{+}-1\right)$ denotes the amount of energy by which $E_{\text {kin }}(t)$ is changed instantaneously when a binary collision is detected. According to the linear spring dashpot model [55], and due to the fact that only kinetic energy in the direction normal to the collision plane is dissipated, the energy loss computed by $\left(b_{j k}^{+}-1\right)$ equals Eq. (2.10):

$$
\left(b_{j k}^{+}-1\right) E_{\mathrm{kin}}(t)=E_{\mathrm{kin}}^{\prime}(t)-E_{\mathrm{kin}}(t)=-\frac{1}{4} m\left(1-e^{2}\right)\left(\boldsymbol{v}_{j k} \cdot \hat{\boldsymbol{r}}_{j k}\right)^{2},
$$

Now, with Eq. (2.13), Eq. (2.12) reads

$$
i \mathcal{L}^{+} E_{\mathrm{kin}}(\boldsymbol{r}, \boldsymbol{v} ; t)=-\frac{1}{4} m\left(1-e^{2}\right) \sum_{j=1}^{N-1} \sum_{k=j+1}^{N}\left|\boldsymbol{v}_{j k} \cdot \hat{\boldsymbol{r}}_{j k}\right| \Theta\left(-\boldsymbol{v}_{j k} \cdot \hat{\boldsymbol{r}}_{j k}\right) \delta\left(r_{j k}-2 a\right)\left(\boldsymbol{v}_{j k} \cdot \hat{\boldsymbol{r}}_{j k}\right)^{2},
$$

where the free streaming part is not considered. According to the detailed steps presented in Appendix A, with Eq. (2.14) we obtain the dissipation rate of a dissipative multi-particle system in the next chapter. 


\subsubsection{Cooling Behavior without Long-range Interactions}

The cooling behavior of a many-body system is determined by the change of kinetic energy per unit time, which is described by the ensemble average of the time derivative of the total kinetic energy. In Appendix A we define all variables and then compute the ensemble average of $d E_{\text {kin }}(t) / d t$ by using the concept of the pseudo-Liouville operator theory. For the dissipation rate in absence of long-range forces,

$$
\begin{aligned}
I^{0}(t):=\frac{d}{d t}\left\langle E_{\mathrm{kin}}\right\rangle(t) & =-8 \pi^{1 / 2} a^{2} N n\left(1-e^{2}\right) g^{0}(2 a) m T_{g}(t)^{3 / 2} \\
& =-\frac{N}{2} f^{0}(t)\left(1-e^{2}\right) m T_{g}(t)
\end{aligned}
$$

with $g^{0}(2 a)$ given in Eq. (A5), we used Eq. (A6) and solved it for the velocity interval $\left.v_{n} \in\right]-$ $\infty, 0]$. This result was derived phenomenologically $[33,76]$ or by means of the pseudo-Liouville operator in spherical coordinates [59], see Appendix A, as by the standard procedure in the literature $[22,11]$. In Eq. (2.15), the negative sign indicates the loss of kinetic energy, and

$$
f^{0}(t)=16 \pi^{1 / 2} a^{2} n g^{0}(2 a) T_{g}(t)^{1 / 2}=12 \nu g^{0}(2 a) T_{g}(t)^{1 / 2} / \sqrt{\pi} a
$$

is the collision rate per particle and unit time, with $T_{g}=2 E_{\mathrm{kin}} /(3 m N)$.

\subsubsection{Cooling Behavior in Presence of Long-range Interactions}

A generalization of the pseudo-Liouville operator theory to long-range interaction potentials consists in a modification of those terms in Eq. (A6) that contribute to the dissipation rate. That is, the pair distribution function $g^{0}(2 a)$, the step-function $\Theta\left(-\sqrt{2} v_{n}\right)$, the Maxwellian $\exp \left(-v_{n}^{2} /\left(2 T_{g}(t)\right)\right)$, the relative velocity $\sqrt{2} v_{n}$ and the squared velocity $v_{n}^{2}$ could change due to the presence of a longrange potential. However, we do not change $g^{0}(2 a)$, the Maxwellian, and the relative velocity, $\sqrt{2} v_{n}$, as discussed in Appendix B. The only terms to be modified are the step-function, which selects the respective range of $v_{n}$, and the energy change due to a collision, i.e., the squared normal relative velocity $v_{n}^{2}$. From Eq. (A6), including the corrections of Appendix B, follows for the repulsive case

$$
\begin{aligned}
I_{\text {rep }}(t) & =-4 \pi a^{2} N n m\left(1-e^{2}\right) g^{0}(2 a)\left(\frac{1}{2 \pi T_{g}(t)}\right)^{1 / 2} \int_{-\infty}^{v_{n, b}} d v_{n} \exp \left(-\frac{v_{n}^{2}}{2 T_{g}(t)}\right)\left|\sqrt{2} v_{n}\right|\left(v_{n}^{2}-v_{n, b}^{2}\right) \\
& =I^{0}(t) \exp \left(-\frac{v_{n, b}^{2}}{2 T_{g}(t)}\right)
\end{aligned}
$$

where the integral limits take into account the energy barrier that has to be overcome when two particles approach. Particle pairs with small relative velocity will not collide, i.e., $v_{n}>v_{n, b}$, with $v_{n, b}<0$. Pairs with higher relative velocity will collide, but the energy loss at contact will be reduced due to the reduced velocity (energy) at contact. 
From Eq. (A6), including the corrections of Appendix B, follows for the attractive case

$$
I_{\text {attr }}(t)=\frac{-4 \pi a^{2} N n m\left(1-e^{2}\right) g^{0}(2 a)}{\left(2 \pi T_{g}(t)\right)^{1 / 2}} \int_{-\infty}^{v_{n, e}} d v_{n} \exp \left(-\frac{v_{n}^{2}}{2 T_{g}(t)}\right)\left|\sqrt{2} v_{n}\right|\left(v_{n}^{2}+v_{n, e}^{2}\right)
$$

analogously to Eq. (2.17), where the modified Heaviside step function is applied, resulting in the upper integral boundary $v_{n}<v_{n, e}$, with $v_{n, e}>0$. Thus, in the case of attractive particles, the energy change due to a collision is larger as compared to the force-free case because the impact velocity of two approaching particles is increased by the attractive escape potential energy. However, this is plausible only for the negative part of the integral, $v_{n}<0$, while the positive part, $v_{n}>0$, has to be discussed further. Splitting the integral accordingly leads to:

$$
\begin{aligned}
I_{\mathrm{attr}}^{ \pm}(t)= & -4 \pi a^{2} N n m\left(1-e^{2}\right) g^{0}(2 a)\left(\frac{1}{2 \pi T_{g}(t)}\right)^{1 / 2} \\
\times & {\left[\int_{0}^{v_{n, e}} d v_{n} \exp \left(-\frac{v_{n}^{2}}{2 T_{g}(t)}\right)\left|\sqrt{2} v_{n}\right|\left( \pm v_{n}^{2}+v_{n, e}^{2}\right)\right.} \\
& \left.+\int_{-\infty}^{0} d v_{n} \exp \left(-\frac{v_{n}^{2}}{2 T_{g}(t)}\right)\left|\sqrt{2} v_{n}\right|\left(v_{n}^{2}+v_{n, e}^{2}\right)\right]
\end{aligned}
$$

with

$$
\begin{aligned}
& I_{\text {attr }}^{+}=I^{0}(t)\left[2-\exp \left(-\frac{v_{n, e}^{2}}{2 T_{g}(t)}\right)+\frac{v_{n, e}^{2}}{T_{g}(t)}\left(1-\exp \left(-\frac{v_{n, e}^{2}}{2 T_{g}(t)}\right)\right)\right] \\
& I_{\text {attr }}^{-}=I^{0}(t)\left[2-\exp \left(-\frac{v_{n, e}^{2}}{2 T_{g}(t)}\right)\right]
\end{aligned}
$$

where the \pm in the first term distinguishes the cases $(+)$ which simply extends the integral, and $(-)$ which accounts for an energy decrease since the velocity of separating particles is reversed.

In the simpler second case, Eq. (2.20), see Ref. [63], the energy term is continuously decreasing with $v_{n}$ increasing from $-\infty$ up to $v_{n, e}$, so that the fastest separating particles are virtually not contributing to the dissipation rate. Nevertheless, in leading order, for small $v_{n, e}^{2} /\left(2 T_{g}(t)\right)$, the two results $I_{\mathrm{attr}}^{ \pm}$are identical, so that we proceed with the simpler Eq. (2.20).

Note that a different point of view is used when the negative or the positive velocities are integrated. In the case of approaching particles, the sample velocity is taken from the Maxwellian when the particles are (infinitely) distant. In contrast, separating particles, when far apart, would never collide, so that the velocity is considered for a pair of particles just after the collision, so that re-collisions are accounted for. Whether this (qualitatively) different point of view should be reflected by a modified $\left|\sqrt{2} v_{n}\right|$ that reflects the re-collision velocity is a possible issue for future studies. 


\section{Simulation}

\subsection{Discrete Element Method}

To check the theoretical predictions in Eqs. (2.17) and (2.20), we will use Molecular Dynamics (MD), also called Discrete Element Method (DEM), where within each time step Newton's equations of motion are solved for all $N$ particles by a simple Verlet integration method. For this, all currently acting forces on each particle have to be known. During a collision between particles $j$ and $k$ with radii $a_{j}=a_{k}=a$ and masses $m_{j}=m_{k}=m$, a linear repulsive contact force, $\boldsymbol{F}_{j}^{\text {short }}$, with a dissipative term $\left(1-e^{2}\right)$ acts as introduced in subsection 2.2.. At the same time, also the long-range forces, $\boldsymbol{F}_{j}^{\text {long }}$, are acting on particle $j$ as introduced in subsection 2.1..

\subsection{Model system}

In order to guarantee a homogeneous particle system, the simulation volume $V=L^{3}$ is provided with periodic boundaries, where particles leaving the boundaries at one side, will immediately enter at the opposite side with unchanged velocity. Therefore, the long-range potential between two particles that feel each other across the periodic boundaries (where one particle is located close to one side and the other close to the opposite side) has to be changed. Two particles "feel" each other across the boundaries and not along their line of sight, dependent on which of the two distances is shorter. Furthermore, one could use a cut-off radius in order to guarantee isotropy, a spherical cutoff radius is introduced so that the total force on particle $j$ is: $\boldsymbol{F}_{j}=\boldsymbol{F}_{j}^{\text {short }}+\boldsymbol{F}_{j}^{\text {long }}\left(r \leq r_{c u t}\right)$ where $r_{c u t}=L / 2$ is half the system length. Different system sizes and either using or not using a force cut-off correction term did not change the results in the situations for which we compared the two options. However, for higher densities and smaller systems (smaller $r_{c u t}$ ) the details of the cut-off are expected to be important. Note that classically a cut-off is only applied to the potential, so that the potential energy barrier is affected directly, whereas in the case of a force-cut-off, the potential energy barrier is modified by the integral of the cut-off force over the approaching path. In the case of small systems or strong potential, caution is required when using either of the options, since the total potential energy and, e.g., the pressure can be different for different force-laws.

\subsection{Algorithmic Implementation}

From the algorithmical side, we implemented a double loop over $N$ particles, and compute the separation length vector, $\boldsymbol{r}_{j k}$, for each pair of particles at every time step. Due to reasons of symmetry and isotropy, we consider only particle pairs whose distances are smaller than $L / 2$, half of one edge of the simulation volume. Thus, if a particle is located in the center of the box, its cut-off sphere then will exactly fit into the simulation box. The computational time cost spent by the algorithm scales with $\mathcal{O}\left(N^{2}\right)$. This is very expensive but can be accepted since we mostly deal with a small number of particles (some $10^{3}$ particles). For much larger $N$, other, much more tedious algorithms must be used in order to reduce the computing time. There are many different approaches for low cost algorithms on the market such as codes that are based on grid or tree 
structures $[27,20,3,32,38,44,80,7]$. Another method e.g., not very different from the cited tree codes, but based on the linked cell neighborhood particle search algorithm [1,67], is described in Refs. [64, 63], i.e., the Hierarchical Linked Cell code.

\section{Results}

This section contains the simulation results for systems without long-range forces as well as for systems with either repulsive or attractive forces in the case of $s=1$.

\subsection{Classical Systems}

Homogeneous many-body systems, where the particles only interact with contact forces as expressed by Eq. (2.11), are in the following referred to as classical systems. The systems considered are spatially homogeneous and the dissipation rate, for $e<1$, is governed by the classical result of Haff, see Eq. (2.15). As long as the dissipative system retains homogeneity, we speak about the homogeneous cooling state (HCS). The HCS is usually a situation that can be met in the beginning of the time evolution of a system - if, of course, the particles were initially homogeneously distributed.

\subsubsection{Elastic systems}

Elastic systems are a special limit case of granular gases because particle interactions are not accompanied by energy dissipation. The system is a thermodynamically closed system and the kinetic energy is conserved. The normal relative velocity just after the collision is the same as the one just before, i.e., $-v_{n}\left(t_{c}\right)=v_{n}(0)$, because the coefficient of normal restitution is unity, $e=1$. In all the equations of section 2.3. the term $\left(1-e^{2}\right)$ vanishes like the dissipation rate

$$
I^{\text {elastic }}(t)=\frac{d}{d t}\left\langle E_{\mathrm{kin}}\right\rangle(t)=\left\langle i \mathcal{L}^{+} E_{\mathrm{kin}}\right\rangle(t)=0
$$

i.e., there is no change of the average total kinetic energy in time. If the gas has settled down for a sufficiently long time, we denote the equilibrium thermal energy as $m T_{g}(\infty)$, where $T_{g}(\infty)$ is the average granular temperature in the steady state. Elastic systems are later used for the preparation of attractive systems - before dissipation is activated. Even though we did not observe a difference between systems prepared elastically with or without the long-range potential, for higher densities and stronger potentials, this might become an issue to be studied further.

\subsubsection{Inelastic systems}

Inelastic systems persistently lose kinetic energy due to successive collisions. For each collision, $v_{n}\left(t_{c}\right)<v_{n}(0)$, since $e<1$, so that according to Eq. (2.15), the dimensionless kinetic energy 

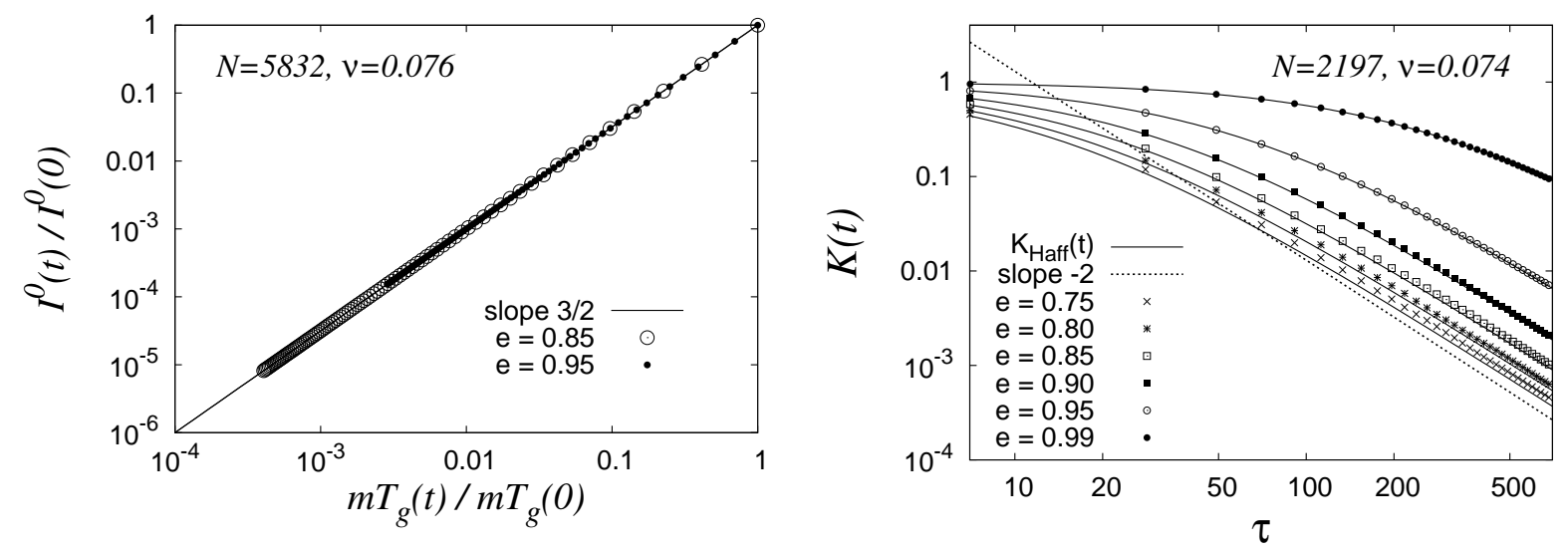

Figure 2: The cooling behavior of inelastic systems can be displayed in two ways: (Left) A double logarithmic representation of the dissipation rate of Eq. (2.15) relative to its initial value, $I^{0}(t) / I^{0}(0)$, plotted against the thermal energy, scaled by its initial value, $m T_{g}(t) / m T_{g}(0)$, for two dissipative simulations with $N=5832, e=0.85$ and 0.95 . The solid line has the slope $3 / 2$. (Right) Double logarithmic representation of the total kinetic energy from Eq. (4.2), relative to its initial value, i.e., $K(t)$, plotted against the dimensionless time, $\tau=t f^{0}(0)$, for different dissipative simulations with $N=2197$ and various $e=0.75 \ldots 0.99$. The dashed line has the slope -2 of the long-time asymptotic behavior. In both panels, symbols represent MD data, lines theory.

decays as

$$
K_{\mathrm{Haff}}(t)=\frac{E_{\mathrm{kin}}(t)}{E_{\mathrm{kin}}(0)}=\frac{1}{\left(1+\frac{1}{6}\left(1-e^{2}\right) \tau\right)^{2}},
$$

where dimensionless time $\tau=t f^{0}(0)$ is scaled by the collision frequency, $f^{0}(0)$, see Eq. (2.16), with temperature, $T_{g}(0)$, at initial time $t=0$, with $E_{\text {kin }}(0)=(3 / 2) N m T_{g}(0) . K_{\text {Haff }}(t)$ thus is the dimensionless kinetic energy of the system according to Haff's law, Eq. (4.2), while we refer to $K(t)$ as the kinetic energy obtained from simulations. In both cases, the kinetic energy is scaled by the (same) initial kinetic energy $E_{\text {kin }}(0)$.

In Fig. 2 the cooling behavior of homogeneous many-body systems with dissipation, but without long-range forces, is displayed in two different ways. The left panel shows the scaled dissipation rate plotted against the scaled actual thermal energy evolving like $T_{g}(t)^{3 / 2}$, as predicted by Eq. (2.15). The right panel shows the scaled total kinetic energy, $K(t)$, plotted against the dimensionless simulation time, $\tau$. As long as the dissipation is weak, $e \approx 1$, the simulation data exactly follow the theoretical prediction from Eq. (4.2). For moderate and strong dissipation, deviations between simulations and theory occur, as evident for the system with $e=0.75$. The deviations stem from inhomogeneities, i.e., the early stages of cluster formation, and from correlations due to strong dissipation - however these issues are beyond the scope of this paper. Qualitatively, dissipation keeps colliding particles together, i.e., they separate considerably slower than they approach each other. This eventually leads to particle assemblies with a locally increased density so that the system is not homogeneous anymore. Below, we will encounter much stronger inhomogeneities 

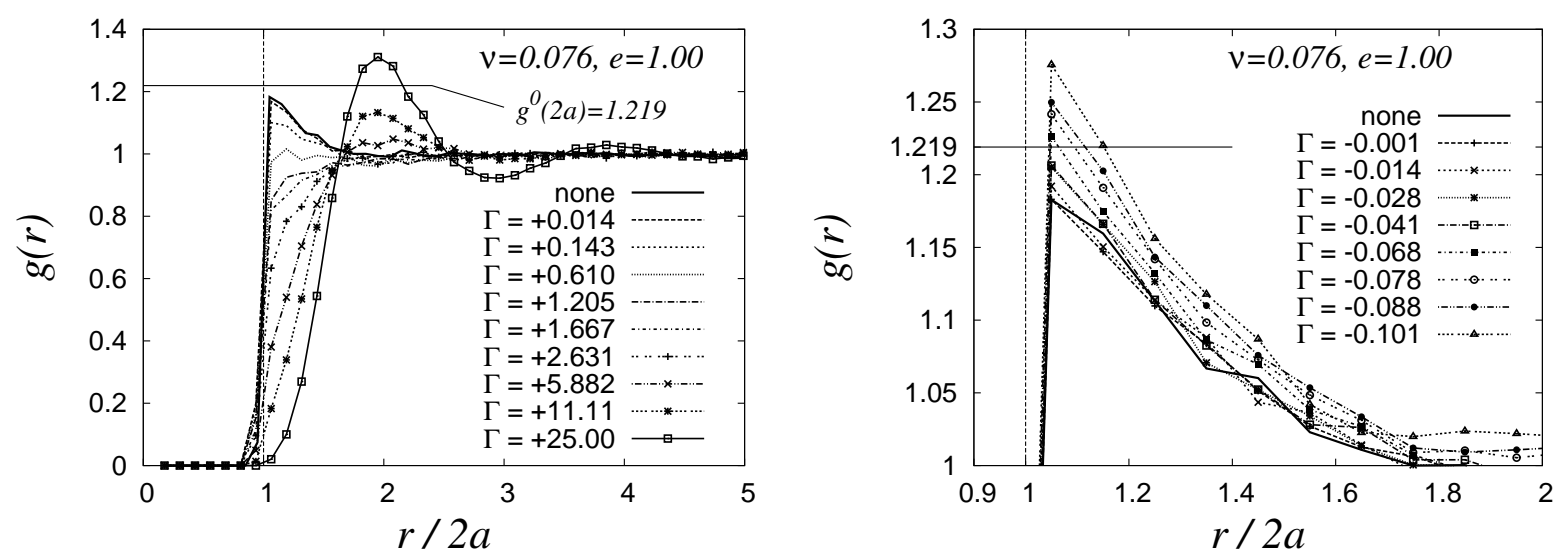

Figure 3: Pair distribution function, $g(r)$, plotted against the particle distance scaled by their diameter, $r / 2 a$, for differently strong coupling, $\Gamma$, in (Left) the repulsive case $(\Gamma>0)$, and (Right) the attractive case $(\Gamma<0)$. Note, that in the right panel, the data are shown only in the range $g(r) \geq 1.0$ and $r / 2 a \geq 0.9$. The line at $g^{0}(2 a)=1.219$ represents the prediction from the Carnahan Starling expression - see main text. The vertical dashed line at $r / 2 a=1$ indicates the excluded volume.

(agglomeration or cluster growth) when strong long-range attractive forces are active together with rather weak dissipation.

\subsection{Systems with long-range potentials}

The collisions and thus the cooling rate of inelastic particle systems are influenced by the longrange interaction potential between the particles: repulsive particles tend to collide less often, whereas attractive particles tend to collide more often than those without a mutual long-range potential. The following subsections deal with the interplay between dissipation and both longrange repulsive and attractive potentials.

\subsubsection{Elastic systems with long-range potentials}

The presence of a long-range potential does not affect the cooling behavior of elastic systems. There is no energy loss for non-dissipative systems, no matter whether these systems are repulsive or attractive. Therefore $\left\langle i \mathcal{L}^{+} E_{\text {kin }}\right\rangle(t)=0$ and after a sufficiently long time of free evolution (some ten collisions/interactions per particle) the system equilibrates with energy $m T_{g}=m T_{g}(\infty)$. (Note however, that the relaxation behavior was not studied quantitatively here. Especially for strong long-range repulsion, the number of collisions will be strongly reduced so that relaxation and equilibration will take place via (long-range) interactions.)

At this point, it is reasonable to introduce the dimensionless long-range potential coupling 
strength

$$
\Gamma:=\frac{v_{\mathrm{cr}}^{2} / 2}{2 T_{g}}=\frac{\Phi(2 a)}{m T_{g}},
$$

i.e., the ratio of the potential energy $\Phi(2 a)$ at contact of two particles, from Eq. (2.8), and the actual thermal fluctuation energy $m T_{g}$ per particle per degree of freedom. Small $\Gamma$ means weak coupling, large values of $\Gamma$ correspond to strong coupling between the long-range potential at contact and the fluctuation kinetic energy in the system. For repulsion and attraction, the coupling strength takes positive and negative values, respectively, where small values of $|\Gamma|$ correspond to weak potentials with respective weak coupling.

In order to investigate how the particles in an elastic system with given $\Gamma$ are spatially distributed, the pair distribution function, $g(r)$, will be measured. It gives the probability to find a neighboring particle $k$ in a distance $r$ from particle $j$, and represents an average over all particle pairs in the system.

For repelling particles, $g(2 a)$ takes smaller values than the analytically known expression for hard spheres: $g^{0}(2 a)=(1-\nu / 2) /(1-\nu)^{3}$, see Refs. [14, 34, 35, 74]. For a given density $\nu=0.076$, the probability at contact, $r / 2 a=1$, is $g^{0}(2 a)=1.219$. In the left panel of Fig. 3 , for small $\Gamma$, the results are identical to the case of no long-range forces (thick solid line) and the value of the pair distribution function at contact is maximal, $g(2 a) \approx 1.2$. The value of $g(2 a)$ decreases with increasing $\Gamma$, and approaches zero for strongest coupling $\Gamma \approx 25$. The pair distribution function approaches unity for large distances $r$, i.e., the system does not show any long-range structures and is homogeneous. For the strongest coupling, $\Gamma \approx 25$, inhomogeneities and correlations reach as far as $r / 2 a=4$. At $r / 2 a \approx 2$, a prominent peak develops, indicating the preferred distance between strongly repulsive neighboring particles, which have practically no chance to collide.

For attractive long-range potentials, see the right panel of Fig. 3, the probability to find two particles in contact increases relative to a system without long-range forces. Strong spatial correlations (corresponding to inhomogeneous systems) are observed for coupling stronger than $\Gamma_{\max } \approx 0.101$, for $r / 2 a \gg 2$ (data not shown), limiting the homogeneous regime to the range of accessible $\Gamma<\Gamma_{\max }$. For attractive systems, dissipation and attraction work together to de-stabilize the homogeneity.

\subsubsection{Inelastic systems with long-range potentials}

In freely cooling dissipative systems, $\Gamma$ cannot be defined as a constant system parameter, since thermal equilibrium is not reached. Nevertheless, the time dependent coupling parameter, $\Gamma(t)=$ $\Phi(2 a) / m T_{g}(t)$, see Eq. (4.3), (absolute value) increases in time with decreasing granular temperature. The energy at contact, $\Phi(2 a)$, becomes more and more important during free cooling. If $\Phi(2 a)$ once has become too strong and the repulsive forces dominate, very few collisions will occur and the granular gas becomes almost elastic. On the other hand, in dissipative attractive systems, a dominating energy $\Phi(2 a)$ leads to inhomogeneities, due to attraction itself and due to the consequently enhanced dissipation. Thus, even when starting with rather small $|\Gamma|$, in repulsive systems, dissipation by collisions is more and more reduced. In contrast, in attractive systems, 

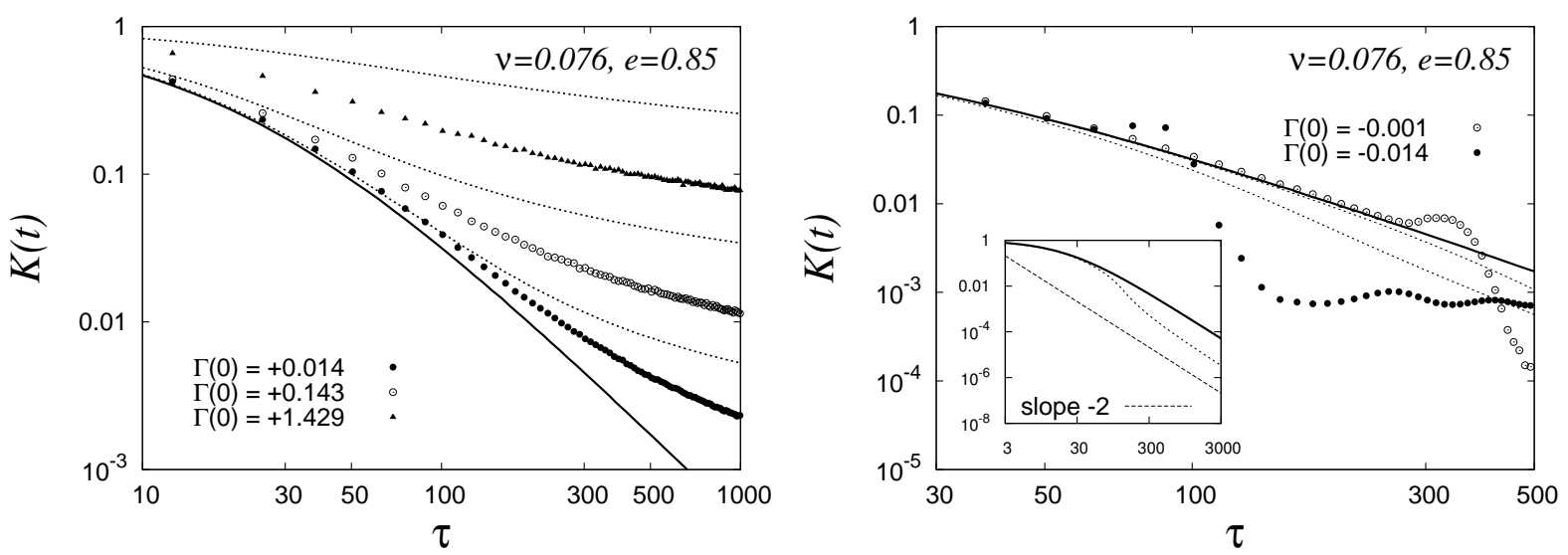

Figure 4: Energy decay in inelastic systems with $e=0.85$ and density $\nu=0.076$, for differently strong initial coupling $\Gamma(0)$. The scaled kinetic energy, $K(t)$, is plotted as function of dimensionless time, $\tau$. In the repulsive case (Left), the solid line corresponds to Eq. (4.2), the dashed lines to solutions of Eq. (2.17) and the symbols are MD data. In the attractive case (Right), the dashed lines correspond to solutions of Eq. (2.20). The inset shows Eqs. (4.2) and (2.20) for a larger time window.

dissipation by collisions is more and more enhanced. In the following, we will use the initial coupling strength, $\Gamma(0)$, as the constant system parameter that defines the importance of the contact potential $\Phi(2 a)$ relative to the initial granular system temperature.

Increasing the potential energy at contact - in both cases, repulsive and attractive as well leads to an earlier onset of the described effects, as can be seen in Fig. 4.

In the left panel the evolution of kinetic energy is shown in the repulsive case. The repulsive interaction hinders the particles from colliding and thus from dissipating kinetic energy. Larger $\Phi(2 a)$ (larger $\Gamma$ ) leads to weaker dissipation (symbols) and also the dissipation rate becomes smaller at later times (due to smaller kinetic energy). The deviation from Haff's law (solid line) sets in earlier for stronger repulsion - which is true for both simulations and theory, however, there is a quantitative difference: Theory under-predicts the dissipation rate.

The right panel shows the same for attractive systems with two differently strong $\Phi(2 a)$. Like for the repulsive systems, also the strongly attractive system (solid circles) deviates earlier from Haff's law than the weakly attractive system (open circles). However, while the theory predicts faster cooling, this is not observed: In simulations of attractive particle systems - for all $\Phi(2 a), e$ and $\nu$ studied - we observe three regimes of evolution: (i) a homogeneous cooling regime, where Haff's law is valid, (ii) an inhomogeneous regime, where the kinetic energy increases strongly before it drops down rapidly on a lower energetic level, and (iii) the agglomeration/clustering regime. Even for much smaller $\Gamma(0)$, due to the ever decreasing velocities of the particles, the system eventually becomes inhomogeneous and finally even forms a single freely rotating agglomerate wherein particles are in permanent contact.

For the above data sets, the theoretical predictions are valid for very short times only (data not shown). Deviations increase with both time and potential energy at contact. In order to identify the 

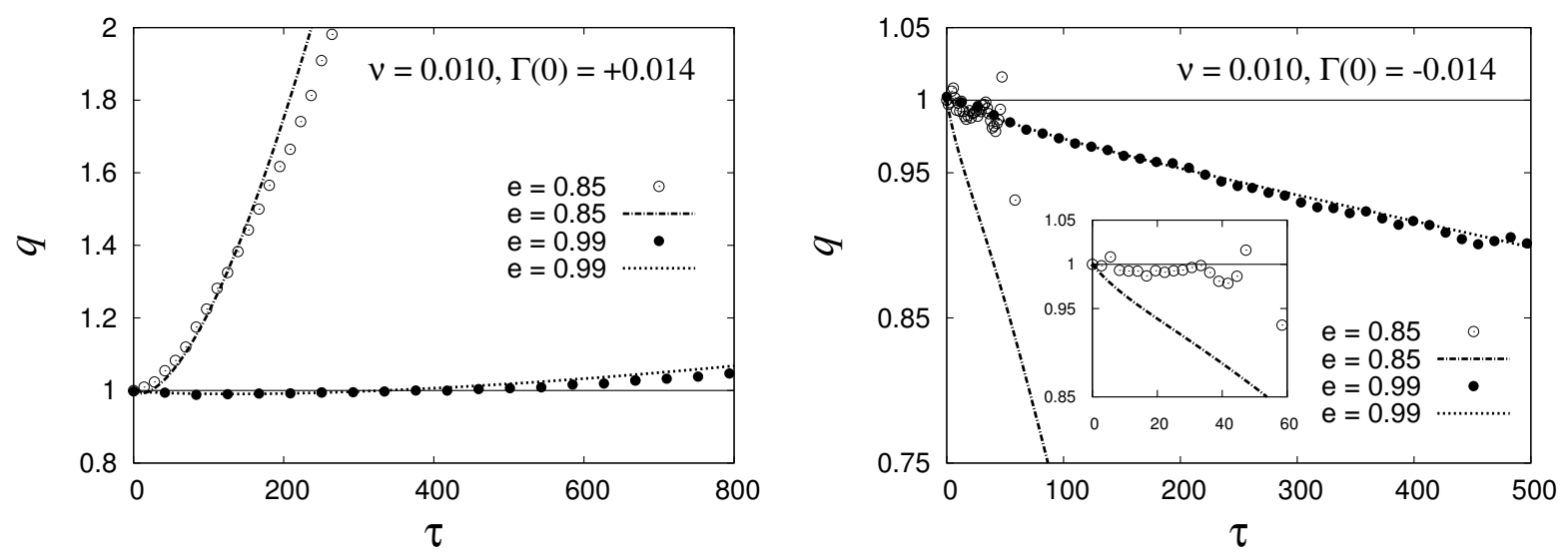

Figure 5: Quality factor $q=K / K_{\text {Haff }}$, with $K_{\text {Haff }}$ from Eq. (4.2), plotted against dimensionless time. For $N=1000$ and $\nu=0.01$, symbols correspond to simulations $\left(q_{\text {data }}\right)$ and lines to the theoretical prediction $\left(q_{\text {theo }}\right)$. Open circles correspond to moderate dissipation $e=0.85$ and solid circles to low dissipation $e=0.99$. (Left) Repulsive simulations with $\Gamma(0)=+0.014$ and (Right) attractive simulations with $\Gamma(0)=-0.014$. The inset shows the simulation data with $e=0.85$, for the first 60 collisions per particle, $\tau=60$.

reasons for this discrepancy, we turn towards (i) lower densities, (ii) weaker dissipation, and (iii) weaker potential energy at contact, in order to make sure that we are in the validity regime of the theory.

\subsubsection{Low density systems with weaker dissipation}

Since the theory in subsection 2.3. only involves pairs of particles, Eqs. (2.17) and (2.20) cannot account for the influence of further neighboring particles on the particle pair of interest. Therefore, the theory should lead to correct predictions only for $\nu \rightarrow 0$, as already shown for the repulsive case by several authors [76, 63]. Considering the attractive case, it was shown in Ref. [63] that the effective escape energy barrier approaches the two-particle escape barrier defined by Eq. (2.8) for $\nu \rightarrow 0$. Furthermore, the analysis must be restricted to the homogeneous cooling state, i.e., the very short time period before agglomeration or clustering sets in.

The predictive power of the theory when applied to dilute dissipative systems is best shown when plotting the "quality factor" that shows how much the two-particle theory deviates from Haff's prediction. For this, a system with very low density $\nu=0.010$ and with an initial coupling strength of $\Gamma(0)= \pm 0.014$ is prepared. The sign "+" denotes the repulsive and "-" the attractive case. The ratio $q$ of the total kinetic energy of the system and the prediction by Haff is either observed from simulations ( $\left.q=q_{\text {data }}\right)$ or by integrating Eqs. (2.17) or (2.20) over time $\left(q=q_{\text {theo }}\right)$. Fig. 5 shows very good agreement between $q_{\text {theory }}$ (dashed lines) and $q_{\text {data }}$ (solid circles) for $e=$ 0.99. The agreement is equally good for both the repulsive (Left) and the attractive case (Right). In contrast, the agreement between $q_{\text {theo }}$ (dashed-dotted lines) and $q_{\text {data }}$ (open circles) for $e=0.85$ is worse. Especially in the attractive case, $q_{\text {data }}$ deviates from $q_{\text {theo }}$ and is much closer to unity 
than to $q_{\text {theo }}$ as shown in the inset of the right panel. Thus, interestingly, for moderately strong dissipation in very low density systems, $q_{\text {data }} \approx 1$, i.e., the system behaves as if there would be no attractive potential: There is no clear sign of the expected increased dissipation due to attractive forces - however, at a certain time $\tau$, the system becomes inhomogeneous .

Having confirmed that the theoretical predictions agree with simulations for low density, weak dissipation and moderate and weak repulsive and attractive potentials, respectively, we next turn to the behavior of the system for larger densities.

\subsection{Towards higher density}

In the last subsection we found that for weak dissipation and low densities the cooling behavior of both repulsive and attractive many-body systems is predicted very well by the two-particle theory. Now, we will study the system for higher densities and, based on these data, empirically extend the theory. The result will be an analytical expression that predicts the cooling rate in the presence of long-range forces also for higher density. This is required for solving the homogeneous cooling regime, where $\Gamma$ is continuously varying with time or - in the future - for inhomogeneous systems, where both density and energy ratio are functions of position and time.

\subsubsection{Empirical expansion of the theory up to moderate densities}

A series of elastic simulations in thermal equilibrium was performed with different densities, excluding the effect of dissipation [63]. This provides the link between the effective energy barrier of the many-particle system and the two-particle (theoretical) prediction $I_{\text {eff }}(t)=H(\nu, \Gamma) I^{0}(t)$, where the dimensionless correction factor $H(\nu, \Gamma) \rightarrow 1$ for $\nu \rightarrow 0$ and $\Gamma \rightarrow 0$, according to the theoretical predictions from Eqs. (2.17) and (2.20). Postulating that $H(\nu \rightarrow 0, \Gamma)$ is given by the theoretical correction terms in Eqs. (2.17) and (2.20), lets us propose the fit-functions $h_{0}, h_{2}$, and $h_{a}$ that depend only on density $\nu$, but not on the energy ratio $\Gamma$. The shape and location of the fit functions is inspired by the numerical results, i.e., no physical meaning should be derived from those $^{\dagger}$. The validity of the assumption that the proposed fit-functions depend only on $\nu$ also for larger $\Gamma$ has to be still validated against advanced theory or improved numerical results with better statistics.

Inserting Eq. (2.8) in Eq. (2.17) using Eq. (4.3), and applying the fit-procedure to the available elastic data for different $\nu$ and $\Gamma$, leads to the new prediction of the dissipation rate:

$$
I(t)=I^{0}(t) \exp \left(-\Gamma(t) h_{0}(\nu)\right)\left(1+a_{2} \Gamma^{2}(t) h_{2}(\nu)\right), \quad \Gamma(t)>0 .
$$

for the repulsive homogeneous cooling state at non-zero densities. Using Eq. (2.20) instead leads

\footnotetext{
${ }^{\dagger}$ More explicitly, the fit procedure is as follows: First fit $h_{0}$ for small $\Gamma$, divide it out, and then fit the quadratic correction, with $a_{2}$ independent of $\nu$, and $h_{2}(\nu)$, for larger $\Gamma$ - where the linear correction $h_{1}$ appears to be negligible. In the attractive case the fits indicate another shape of the correction function, $h_{a}$, only correcting the exponential since the available range of $\Gamma$ is much smaller. The quality of the fits is in the range of approximately five per-cent [63].
} 
to the new prediction for the dissipation rate:

$$
I(t)=I^{0}(t)\left[2-\exp \left(\Gamma(t) h_{a}(\nu)\right)\right], \quad \Gamma(t)<0 .
$$

for the attractive case [63], in the homogeneous cooling state for non-zero densities.

In the above equations, $a_{2}$ is a constant and $h_{0}(\nu), h_{2}(\nu)$ and $h_{a}(\nu)$ depend on $\nu$ alone and all tend to $h(\nu) \rightarrow 1$ for $\nu \rightarrow 0$. These density functions were obtained in Ref. [63] by fitting the scaled collision rates, $f(t) / f^{0}(t)$, as:

$$
\begin{aligned}
& h_{0}(\nu)=\exp \left(-b_{0} \nu^{c_{0}}\right), \\
& h_{2}(\nu)=\exp \left(-b_{2} \nu^{c_{2}}\right), \text { and } \\
& h_{a}(\nu)=1+a_{1} \nu,
\end{aligned}
$$

where $b_{0}=5.468, c_{0}=0.628, a_{2}=0.065, b_{2}=9.002, c_{2}=0.790$, and $a_{1}=-4.419$ are the fit parameters. Note that these corrections were obtained from elastic systems with the densities $\nu=0.010,0.038,0.076,0.114$, and 0.152, for $0<\Gamma<12$ (repulsive) and $-0.1<\Gamma<0$ (attractive). Establishing the predictions for higher densities $\nu>0.152$ is beyond the scope of this study - even though the present results allow for an extrapolation for larger densities, the quality of which has to be checked in the future.

Furthermore, note that the major result of this paper, the corrected dissipation rates in Eqs. (4.4) and (4.5) require an additional multiplicative correction term. Due to the soft contact interaction, the dissipation rate is reduced by approximately $\exp \left(-2 \tau_{c}\right)$, where $\tau_{c}=t_{c} f(t)$ is the ratio of contact duration to time between collisions. This is because multi-particle contacts are probable, especially for large $t_{c}$, large densities and high velocities, as discssued in detail in Refs. [56, 53, $50,52]$. For the examples shown below this correction is weak, so that we do not apply it here, but just refer to the previous work.

\subsubsection{Systems with considerable density and dissipation}

In order to challenge the predictive value of the empirically obtained correction, $H(\nu, \Gamma)$, we compare it to simulations of repulsive and attractive systems with moderate densities and rather strong dissipation. Fig. 6 shows the linear-logarithmic plot of $K(t)$ as a function of $\tau$ for a repulsive (left) and attractive dissipative gas (right panel). As reference, also Haff's theory (thin solid line) and the dilute limit solution (dashed line) of Eqs. (2.17) and (2.20) are plotted. These solutions are improved by considering the empirically obtained quantity $H(\nu, \Gamma)$ and are represented by the thick solid line close to the symbols. Even though the correction is derived from simulations without dissipation, there is an improvement of the predictions for both the repulsive and the attractive case (compare dashed with thick solid line as compared to the symbols). For large times, however, the difference between data and the empirically extended theory becomes larger and is very likely due to the rather strong dissipation. 

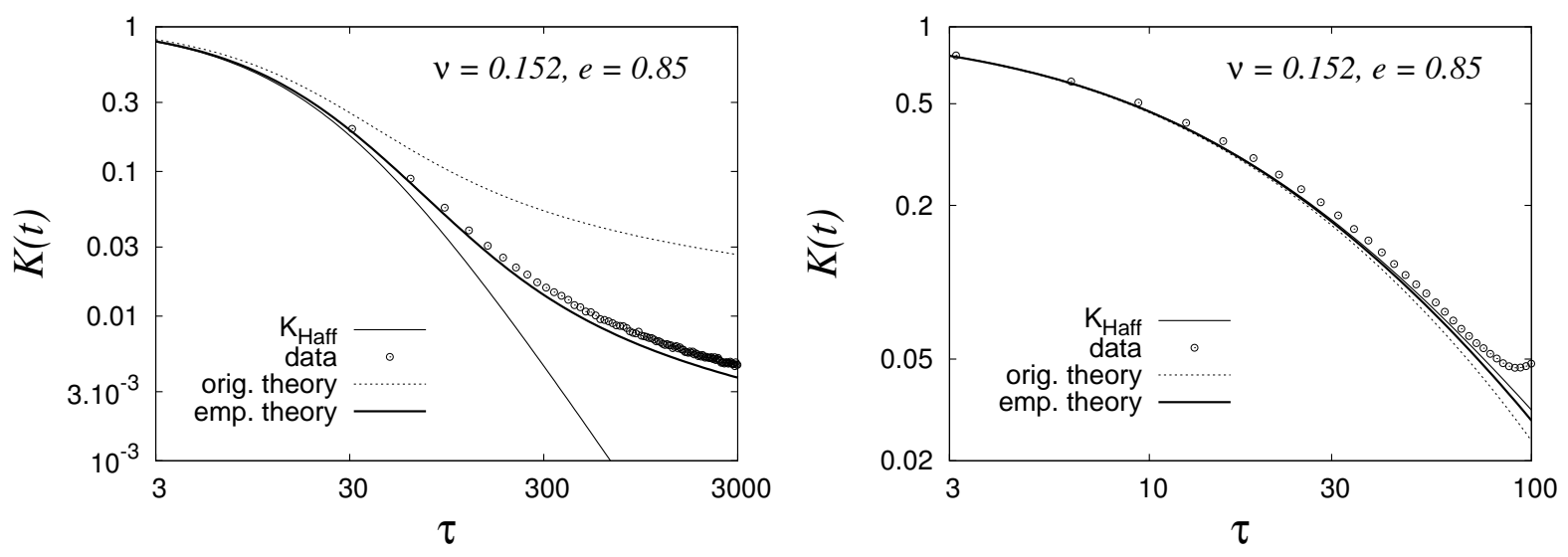

Figure 6: Scaled kinetic energy, $K(t)$, plotted against the scaled time, $\tau$, for systems with $N=$ 1000, $\nu=0.152$, and $e=0.85$. The thin solid lines correspond to Haff's Eq. (4.2), symbols are simulation data. (Left) The repulsive case with $\Gamma(0)=+0.14$. The dashed line denotes the original theory Eq. (2.17), the thick solid line the empirically modified theory Eq. (4.4) with $h_{0}(0.152)=0.187$ and $h_{2}(0.152)=0.131$. (Right) The attractive case with $\Gamma(0)=-0.014$. The dashed line denotes the original theory Eq. (2.20), the thick solid line the empirically modified theory Eq. (4.5) with $h_{a}(0.152)=0.328$.

In the attractive case (right panel) at moderate density and dissipation, data show Haff-like behavior, i.e., symbols are close to Haff's prediction. The reason for the discrepancy between the data set and Haff's theory is due to the formation of weak inhomogeneities when rather strong dissipation is active - as already stated in subsection 4.1.2..

Both for repulsive and attractive systems, the dilute theory overestimates the coupling strength. This leads in the repulsive case to less, and in the attractive case to more, collisions relative to the corresponding simulations. In the first case, a too small energy loss (the dashed line evolves above the symbols), whereas in the latter case, a too large energy loss is predicted (the dashed line evolves below the symbols).

Generally, at moderate densities and rather strong dissipation, the cooling behavior can be predicted well for homogeneous repulsive particle systems by the empirical extension of the twoparticle theory. Interestingly, for attractive particle systems with considerable dissipation, Haff's theory remains a good prediction.

\subsection{Long-time cooling behavior of repulsive systems}

When a dissipative, repulsive system cools down for sufficiently long time, $\Phi(2 a)$ will become larger as compared to the fluctuating kinetic energy, $m T_{g}(\tau)$. A critical dimensionless time, $\tau_{c r}$, can be defined, when the actual thermal energy equals the potential energy barrier, i.e., when $\Gamma\left(\tau_{c r}\right)=1$. For large $\tau_{c r}$, which is equivalent to small $\Gamma(0)$, from Haff's law Eq. (4.2) follows the 

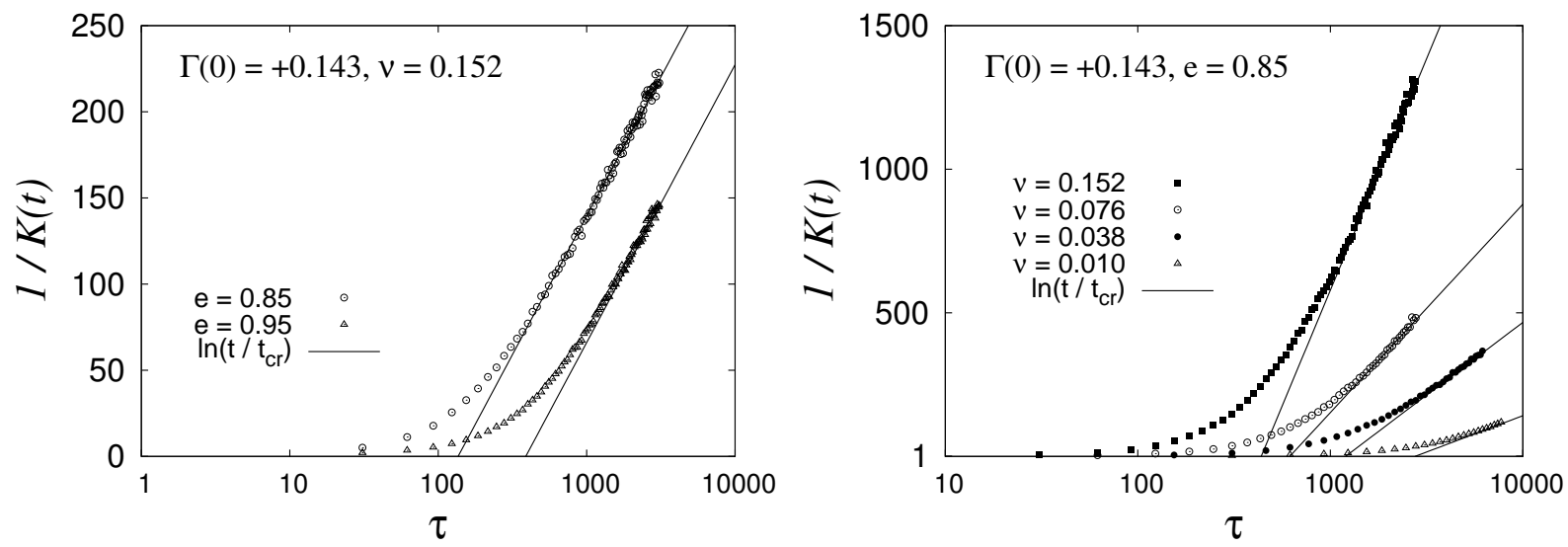

Figure 7: Asymptotic long-time cooling behavior of dissipative repulsive systems. The inverse of $K(t)$ is plotted as a function of dimensionless time $\tau$. Symbols are data, straight lines correspond to Eq. (4.10) in the limit $\tau \rightarrow \infty$. (Left) Fixed density and coupling strength with different dissipation, e. (Right) Fixed dissipation and coupling strength for different densities, $\nu$.

estimate:

$$
\tau_{c r} \approx \frac{2}{1-e^{2}} \sqrt{\frac{1}{\Gamma(0)}} .
$$

In Ref. [76], Eq. (2.17) is solved asymptotically for $\tau \rightarrow \infty$ and the inverse energy is approximated by a logarithm:

$$
\frac{1}{K(t)} \propto \ln \left(\frac{\tau}{\tau_{c r}}\right),
$$

which expresses the asymptotic cooling behavior of a dissipative, repulsive granular system for large times. In Fig. 7 inverse kinetic energy is plotted against logarithmic time for inelastic repulsive systems. In the left panel, cooling systems with different dissipation for a given (high) density $\nu=0.152$ are shown. In the right panel, systems for different densities and with a given (strong) dissipation $e=0.85$ are shown. Systems of the same density but different dissipation reach asymptotes with about the same slope, whereas different densities lead to different slopes. The asymptotic behavior is shown for large $\tau$ and is indicated by the the straight solid lines that obey Eq. (4.10). It is reached earlier in time for faster cooling systems, which can be achieved by stronger dissipation or higher density - as confirmed by the simulations.

Logarithmic long-time cooling behavior, as expressed by Eq. (4.10), is also observed for other systems that evolve towards a more elastic regime. A qualitatively similar cooling behavior - without the presence of long-range interaction forces - is reported for systems with velocity-dependent dissipation, $e=e\left(v_{n}\right)$. When the dissipated amount of kinetic energy in a particle collision is a decreasing function of the impact velocity $[73,12]$, the system approaches the elastic limit, $e \rightarrow 1$, for large $\tau$. The so-called velocity cut-off model, which has a constant $e$, but sets $e=1$ for all collisions below a certain cut-off velocity, can be analytically solved, similar in spirit to the theory 


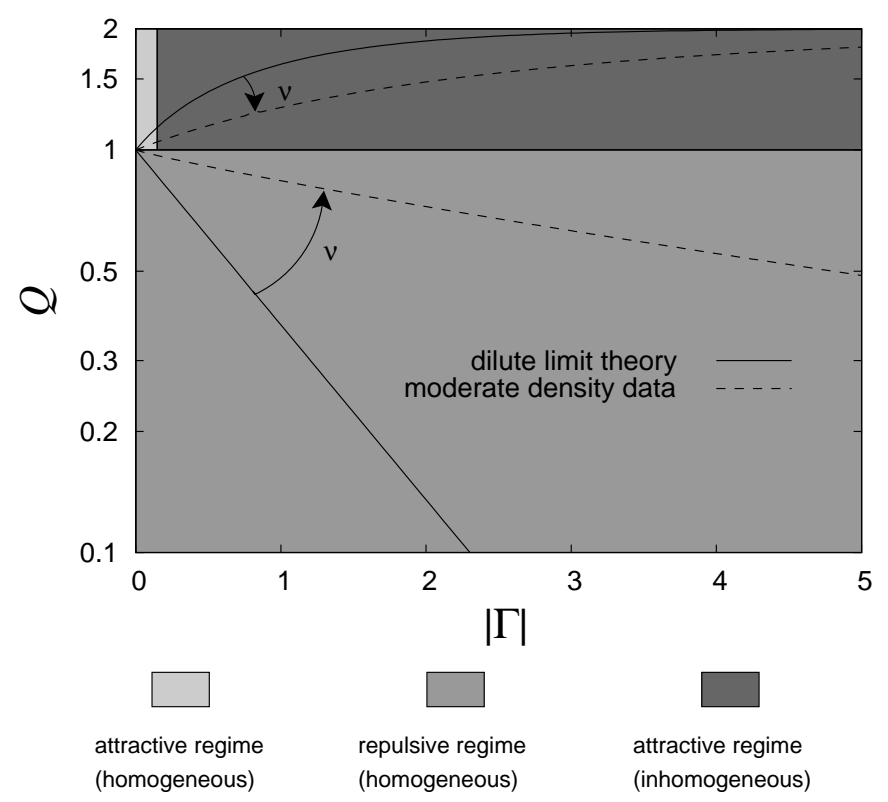

Figure 8: The quality factor, $Q=I / I^{0}$, i.e., the correction to the classical dissipation rate, $I^{0}$, without long range interactions, plotted as a function of the coupling strength, $|\Gamma|$, for both the repulsive and the attractive regime. $Q=1$ corresponds to the classical (Haff) cooling regime, the solid lines represent the (low-density) theory, Eqs. (2.17), (2.20), and the dashed lines give the empirical correction for finite density, Eqs. (4.4), (4.5), for the maximal density $\nu=0.152$.

presented in this study. The dissipation rate is then similar to the one expressed by Eq. (2.17), see Ref. [53].

\section{Conclusion}

Three-dimensional soft-sphere Molecular Dynamics simulations with pair-wise summation of forces due to the $(1 / r)$ long-range interaction potentials were carried out. The two-particle pseudoLiouville operator theory for hard spheres is corrected/extended for both repulsive and attractive long-range interactions and compared to the simulation results.

For very low densities and weak dissipation, the theory predicts very well the simulation results. For repulsive forces, theory recovers the results of Scheffler and Wolf [76], a reduced dissipation due to repulsion. The reduced dissipation is accounted for by a Boltzmann like exponential correction factor, $\exp (-\Gamma)$, in the dissipation rate, which decays exponentially with the coupling strength $\Gamma$ that relates the contact potential energy to the fluctuation kinetic energy (granular temperature). For attractive forces, our theory predicts enhanced dissipation, however, with a different analytical form of the correction factor, $2-\exp (-\Gamma)$. Note that in the limit of small coupling strength, both the repulsive and attractive regime are expressed by the same (leading order) functional form $1-\Gamma$, with $\Gamma>0$ for repulsive interactions and $\Gamma<0$ for attractive interactions. 
However, for higher densities and/or stronger dissipation, the cooling behavior is not well described by the theory. In the repulsive case, dissipation is strongly under-predicted, but qualitatively described well. In the attractive case, it is even qualitatively predicted badly: Simulations show no or even slightly reduced dissipation, whereas theory predicts slightly enhanced dissipation. The theoretical results for the correction factor to the dissipation rate are displayed as solid lines in the phase-diagram in Fig. 8.

Therefore, we generalized the low-density pseudo-Liouville operator theory to higher densities by fits to numerical simulations of systems with various strengths of the interaction potential but without dissipation. In the range $0.010 \leq \nu \leq 0.152$ we obtained empirical correction functions that depend on the density only. The results for the empirical correction to the dissipation rate (needed for non-zero density) are displayed as dashed lines in Fig. 8. Inserting this empirical result into the collision rate in fact improves very much the quantitative predictive power of the theory. Note that repulsive potentials stabilize the homogeneous system, whereas attractive potentials facilitate the system becoming inhomogeneous, as indicated by the two grey-scales in the attractive regime in Fig. 8. Only for very weak attractive potentials, the homogeneous cooling state is stable and the theory is able to predict the systems cooling behavior.

Finally, we observe that repulsive dissipative systems become elastic for long times: The longtime energy decay is proportional to the logarithm of time, which we were able to confirm by simulations of systems with different dissipation and density. This behavior is similar to that of systems with a velocity dependent restitution coefficient, where slower collision velocities involve less dissipation than higher ones.

Future studies should address the hydrodynamic stability of the present system and also (i) higher dissipation, (ii) higher density, as well as (iii) the case of repulsive and attractive potentials mixed. (i) For higher density and repulsion, the formation of plasma-crystal like structures is expected for very low mass density due to the long range forces. (ii) For higher densities and attraction, the system resembles inter-stellar dust-clouds or even larger scale astrophysical systems where both dissipation and attractive potentials are relevant - their interplay is not sufficiently understood at present. (iii) In realistic systems of dry particles, due to tribo-charging, positive as well as negative charges will co-exist, so that the present study has to be extended towards this interesting regime too.

\section{A Ensemble Averages}

Since we deal with many-body systems, we are interested in ensemble averages of observables. A representative observable of the cooling behavior of the system is the energy dissipation rate, i.e., the time derivative of the total kinetic energy, and its ensemble average is expressed as

$$
\frac{d}{d t}\left\langle E_{\mathrm{kin}}\right\rangle(t)=\int \prod_{l=1}^{N} d \boldsymbol{r}_{l} d \boldsymbol{v}_{l} \rho(\{\boldsymbol{r}\},\{\boldsymbol{v}\} ; 0) \frac{d}{d t} E_{\mathrm{kin}}(\{\boldsymbol{r}\},\{\boldsymbol{v}\} ; t),
$$

where $\rho(\{\boldsymbol{r}\},\{\boldsymbol{v}\} ; 0)$ is the phase space distribution function at initial time $t=0$, while the observable, $(d / d t) E_{\text {kin }}(\{\boldsymbol{r}\},\{\boldsymbol{v}\} ; t)$, has already been propagated to time $t$. The sets $\{\boldsymbol{r}\}$ and 
$\{\boldsymbol{v}\}$ are the $N$ single particles' positions and velocities. Since the time evolution of an observable can be expressed by the pseudo-Liouville operator acting on the observable, we can write $(d / d t) E_{\text {kin }}(\{\boldsymbol{r}\},\{\boldsymbol{v}\} ; t)=i \mathcal{L}^{+} E_{\text {kin }}(\{\boldsymbol{r}\},\{\boldsymbol{v}\} ; t)$. So, Eq. (A1) can then be rewritten as

$$
\frac{d}{d t}\left\langle E_{\text {kin }}\right\rangle(t)=\int \prod_{l=1}^{N} d \boldsymbol{r}_{l} d \boldsymbol{v}_{l} \rho(\{\boldsymbol{r}\},\{\boldsymbol{v}\} ; 0) i \mathcal{L}^{+} E_{\text {kin }}(\{\boldsymbol{r}\},\{\boldsymbol{v}\} ; 0)=\left\langle i \mathcal{L}^{+} E_{\text {kin }}\right\rangle(t)
$$

Using the pseudo-Liouville operator of Eq. (2.14), the integral in Eq. (A2) has to be solved in order to obtain the ensemble average $(d / d t)\left\langle E_{\text {kin }}\right\rangle(t)$. In the following, we will solve this integral in a standard way $[55,59]$ but skip most steps and detail the changes made in order to include longrange forces [63].

Using $\rho(\{\boldsymbol{r}\},\{\boldsymbol{v}\} ; 0)$ with the assumption of homogeneity, isotropy, a Maxwellian velocity distribution of the particles $[59,63]$ and molecular chaos in particular, allows for separating the coordinate part from the velocity part in $\rho(\{\boldsymbol{r}\},\{\boldsymbol{v}\} ; 0)$. Since the binary collision operator $b_{12}^{+}$ in Eq. (2.12) acts on two out of the $N$ particles, one can introduce the two-particle distribution function, $g^{0}\left(\boldsymbol{r}_{1}-\boldsymbol{r}_{2}\right)$, where the superscript " 0 " denotes the force-free case. Integration over the remaining $N-2$ spatial particle coordinates and velocities leads to an expression for the ensemble average that is dependent on the coordinates and velocities of the two particles only. With the interaction part of the pseudo-Liouville operator in Eq. (2.14) follows

$$
\begin{aligned}
& \frac{d}{d t}\left\langle E_{\text {kin }}\right\rangle(t)=-\frac{1}{4} \frac{N^{2}}{V^{2}}\left(\frac{1}{2 \pi T_{g}(t)}\right)^{3} m\left(1-e^{2}\right) \\
& \times \int d \boldsymbol{R} d \boldsymbol{r} d \boldsymbol{V} d \boldsymbol{v} g^{0}(\boldsymbol{r}) \exp \left(-\frac{\boldsymbol{v}^{2}+\boldsymbol{V}^{2}}{2 T_{g}(t)}\right)|\sqrt{2} \boldsymbol{v} \cdot \hat{\boldsymbol{r}}| \Theta(-\sqrt{2} \boldsymbol{v} \cdot \hat{\boldsymbol{r}}) \delta(\boldsymbol{r}-2 a \hat{\boldsymbol{r}})(\boldsymbol{v} \cdot \hat{\boldsymbol{r}})^{2}
\end{aligned}
$$

where $\boldsymbol{R}=\boldsymbol{R}_{1}+\boldsymbol{R}_{2}, \boldsymbol{r}=\boldsymbol{r}_{1}-\boldsymbol{r}_{2}$ and $\boldsymbol{V}=(1 / \sqrt{2})\left(\boldsymbol{v}_{1}+\boldsymbol{v}_{2}\right), \boldsymbol{v}=(1 / \sqrt{2})\left(\boldsymbol{v}_{1}-\boldsymbol{v}_{2}\right)$. With $n=N / V$ and integration over $\boldsymbol{R}, \boldsymbol{r}$ and $\boldsymbol{V}$, Eq. (A4) gives

$$
\begin{aligned}
\frac{d}{d t}\left\langle E_{\text {kin }}\right\rangle(t)= & -4 \pi a^{2} N n m\left(1-e^{2}\right) g^{0}(2 a)\left(\frac{1}{2 \pi T_{g}(t)}\right)^{3 / 2} \\
& \times \int d \boldsymbol{v} \exp \left(-\frac{\boldsymbol{v}^{2}}{2 T_{g}(t)}\right)|\sqrt{2} \boldsymbol{v} \cdot \hat{\boldsymbol{r}}| \Theta(-\sqrt{2} \boldsymbol{v} \cdot \hat{\boldsymbol{r}})(\boldsymbol{v} \cdot \hat{\boldsymbol{r}})^{2}
\end{aligned}
$$

with the pair distribution function at contact $(\boldsymbol{r}=2 a \hat{\boldsymbol{r}})$ without considering long-range forces $[74,14,34,35]$ :

$$
g^{0}(2 a)=\frac{1-\nu / 2}{(1-\nu)^{3}} .
$$

In order to solve the velocity integral in Eq. (A4), we do not use spherical coordinates as usual but rotate a Cartesian system such that the centre-to-centre-vector, $\hat{r}$, defines one axis. We then are able to split up the relative velocity vector like in subsection 2.1. as $\boldsymbol{v}^{2}=(\boldsymbol{v} \cdot \hat{\boldsymbol{r}})^{2}+(\boldsymbol{v} \cdot \hat{\boldsymbol{t}})^{2}+(\boldsymbol{v} \cdot \hat{\boldsymbol{s}})^{2}$, where the normal, tangential and out-of-plane unit vectors $\hat{\boldsymbol{r}}, \hat{\boldsymbol{t}}, \hat{\boldsymbol{s}}$ are perpendicular to each other 
and $\boldsymbol{v} \cdot \hat{\boldsymbol{s}}=0$. Integration over $\boldsymbol{v} \cdot \hat{\boldsymbol{t}}$ and $\boldsymbol{v} \cdot \hat{\boldsymbol{s}}$ provides

$$
\begin{aligned}
\frac{d}{d t}\left\langle E_{\text {kin }}\right\rangle(t)= & -4 \pi a^{2} N n m\left(1-e^{2}\right) g^{0}(2 a)\left(\frac{1}{2 \pi T_{g}(t)}\right)^{1 / 2} \\
& \times \int_{-\infty}^{+\infty} d v_{n} \exp \left(-\frac{v_{n}^{2}}{2 T_{g}(t)}\right)\left|\sqrt{2} v_{n}\right| \Theta\left(-\sqrt{2} v_{n}\right) v_{n}^{2},
\end{aligned}
$$

leaving the normal relative impact velocity, $v_{n}=\boldsymbol{v} \cdot \hat{\boldsymbol{r}}$, as the remaining integration variable. The step function selects velocities from the interval $[-\infty, 0]$ and can be removed if we change the integration limits accordingly. We can consider the cases with and without long-range interactions by taking corrections of some terms in Eq. (A6) into account. These terms are discussed in the following and corrections are applied where necessary.

\section{B Corrections}

The pseudo-Liouville operator selects only those particle pairs from all $N(N-1) / 2$ pairs in the system that $d o$ collide. For the case without long-range interactions, Eq. (A6) has simply to be solved for the interval $\left.\left.v_{n} \in\right]-\infty, 0\right]$. In case of long-range interactions some terms in Eq. (A6) are corrected before the integral is carried out. Imagine, that in the dilute limit, two particles are approaching from far apart, i.e., the free path, $l$, between collisions is large and the long-range potential is relatively weak.

\section{The pair distribution function at contact $g^{0}(2 a)$}

The pair distribution function at contact gives the average probability to find two particles with a separation length of $\boldsymbol{r}=2 a \hat{\boldsymbol{r}}$. The contact probability is decreased if the particles feel a repulsive potential and increased if the particles are attractive. That means, the effective excluded volume is changed accordingly, which leads to an effective particle radius. As it is shown in [75, 76, 63] and as we will show a-posteriori, the modification of the pair distribution function at contact is described by the correction factors we found when solving the ensemble averages for the dissipation rate in presence of a long-range potential:

$$
\begin{aligned}
& \frac{g(2 a)}{g^{0}(2 a)}=\frac{f(t)}{f^{0}(t)}=\frac{I(t)}{I^{0}(t)}=\exp \left(-\frac{\Phi_{\mathrm{eff}}}{m T_{g}(t)}\right) \quad \text { for } \quad \Phi_{\mathrm{eff}}>0, \\
& \frac{g(2 a)}{g^{0}(2 a)}=\frac{f(t)}{f^{0}(t)}=\frac{I(t)}{I^{0}(t)}=2-\exp \left(-\frac{\Phi_{\mathrm{eff}}}{m T_{g}(t)}\right) \quad \text { for } \quad \Phi_{\mathrm{eff}}<0 .
\end{aligned}
$$

$g(2 a)$ considers long-range interactions and is displayed in Fig. 3, in the left panel for repulsive, in the right panel for attractive forces. The superscript " 0 " denotes the respective quantities without the consideration of long-range interactions. So, the right-hand sides of Eq. (B1) are already the corrections of $g^{0}(2 a)$, which thus needs not to be modified in Eq. (A6). 


\section{The selection term, i.e., the Heaviside step-function $\Theta\left(-\sqrt{2} v_{n}\right)$}

The step function in Eq. (A6) selects only particles that approach each other, i.e., it takes normal relative velocities into account that are negative. In presence of repulsive interactions, the step function should select from the interval $\left.]-\infty, v_{n, b}\right]$, where $v_{n, b}<0$. This leads to the integral boundaries $\left.]-\infty, v_{n, b}\right]$ and

$$
\Theta\left(-\sqrt{2} v_{n}\right) \rightarrow \Theta\left(-\sqrt{2}\left(v_{n}-v_{n, b}\right)\right) .
$$

In contrast, for attractive interactions, the step function not only considers approaching particles from the interval $]-\infty, 0$ ] but also separating particles from the interval $\left.] 0, v_{n, e}\right]$, where $v_{n, e}>0$. The selection of the step function leads to the integral boundaries $\left.]-\infty, v_{n, e}\right]$ and

$$
\Theta\left(-\sqrt{2} v_{n}\right) \rightarrow \Theta\left(-\sqrt{2}\left(v_{n}-v_{n, e}\right)\right)
$$

\section{The Maxwellian velocity distribution term $\exp \left(-v_{n}^{2} /\left(m T_{g}(t)\right)\right)$}

The exponential in Eq. (A6) comes from the assumption of a Maxwellian distribution of the normal relative velocities. Since the selection of normal relative velocities from the Maxwellian due to the presence of long-range interactions is already done by the step-function, the Maxwellian is not changed. This corresponds to the assumption of an undisturbed Maxwellian for distant particles, before they come close to each other.

\section{The relative velocity term $\left|\sqrt{2} v_{n}\right|$}

The term $\left|\sqrt{2} v_{n}\right|$ determines the collision rate which is dependent on $v_{n}$. The larger $v_{n}$, the more collisions do occur per particle and unit time because the probability for collisions increases with increasing velocity. In the following we will estimate whether it is important to correct this term in the presence of long-range interactions for low densities. According to Eq. (2.5), the kinetic energy is dependent on $r$ and reads

$$
\frac{1}{4} m v^{2}=\frac{1}{4} m\left(v_{n \infty}\right)^{2}-\Phi(r)
$$

where $v=v(r)$ is the relative impact velocity that is distance dependent for a given (initial) $v_{n \infty}$. The time between two collisions, i.e., the inverse collision rate, depends on $\Phi(r)$. The path length, $l$, which both particles travel from infinity until the collision takes place, is obtained by integration of $r$ over the interval $[l+2 a, 2 a]$ :

$$
t_{l}\left(v_{n \infty}, \Phi\right)=\int_{l+2 a}^{2 a} \frac{d r}{v(r)}=\int_{l+2 a}^{2 a} \frac{d r}{(-1) \sqrt{v_{n \infty}^{2}-4 \Phi(r) / m}} \stackrel{(2.1)}{=} \frac{1}{v_{n \infty}} \int_{l+2 a}^{2 a} \frac{d r}{\sqrt{1+4 \alpha / m r v_{n \infty}^{2}}}
$$

where the parameters $v_{n \infty}$ and $\Phi$ (with $s=1$ ) determine the $r$-dependence of the collision time. Here, $d r<0$ because the distance decreases with increasing time, and $v(r)$ and $v_{n \infty}$ are negative 
because of the sign-convention for approaching particles. Integration over the whole range of separation leads to the time span both particles need until they collide. For very weak potentials, $\Phi(r) \ll(1 / 4) m v_{n \infty}^{2}$, we can expand Eq. (B3) to the second order

$$
\begin{aligned}
t_{l}\left(v_{n \infty}, \Phi\right) & \approx \frac{1}{v_{n \infty}} \int_{l+2 a}^{2 a} d r\left(1-\frac{1}{2} \frac{4 \alpha}{m v_{n \infty}^{2} r}\right)=\frac{2 a}{v_{n \infty}} \int_{(l / 2 a)+1}^{1} d r^{\prime}\left(1-\frac{2 \alpha}{m v_{n \infty}^{2}(2 a) r^{\prime}}\right) \\
& =\frac{l}{\left|v_{n \infty}\right|}-\frac{2 \alpha}{m\left|v_{n \infty}\right|^{3}} \ln \left(\frac{l}{2 a}+1\right) .
\end{aligned}
$$

The right-hand side is obtained by substituting $r=2 a r^{\prime}$ and $d r=2 a d r^{\prime}$. The case $\alpha=0$ leads to the collision time in absence of long-range forces: $t_{l}^{0}=t_{l}\left(v_{n \infty}, 0\right)=l /\left|v_{n \infty}\right|$. For repulsive interactions $(\alpha<0)$, the time span is increased because particles are decelerated when approaching, whereas for attractive interactions $(\alpha>0), t_{l}$ is decreased because particles are accelerated when approaching.

Now, we can set

$$
\begin{aligned}
|\sqrt{2} v(r)| & =\left|\frac{\sqrt{2} l}{t_{l}\left(v_{n \infty}, \Phi\right)}\right| \approx\left|\sqrt{2} l\left(\frac{l}{\left|v_{n \infty}\right|}-\frac{2 \alpha}{m\left|v_{n \infty}\right|^{3}} \ln \left(\frac{l}{2 a}+1\right)\right)^{-1}\right| \\
& \approx\left|\sqrt{2} v_{n \infty}\right|\left(1+\frac{2 \alpha}{m l v_{n \infty}^{2}} \ln \left(\frac{l}{2 a}+1\right)\right)^{-1}
\end{aligned}
$$

valid for $\Phi(r) \ll(1 / 4) m v_{n \infty}^{2}$. Even for rather strong potentials, in the dilute limit, where $\nu \rightarrow 0$ and $l \rightarrow \infty$, we have

$$
|\sqrt{2} v(r)| \rightarrow\left|\sqrt{2} v_{n \infty}\right|
$$

because $1 / l$ decreases faster than $\ln (l /(2 a)+1)$ increases. This means, with other words, for low densities, most of the time between two collisions the velocity of both particles is practically unchanged by the presence of the long-range potential. Thus, in the first-order approach, the term $\left|\sqrt{2} v_{n \infty}\right|$ in Eq. (A6) is not modified. Note, that also the free streaming part of section 2.3. is not modified for the same reasons.

More advanced theory [21, 23], however, predicts a dependence of the collision rate on the relative velocity with a power law $\left|v_{n}\right|^{\psi}$, with $\psi=1-2(\mathcal{D}-1) / s$. This could require additional corrections of the relative velocity term, which we neglect here for the sake of brevity.

\section{The energy dissipation term $v_{n}^{2}$}

Eq. (2.13) gives the difference between the total kinetic energy of the system after one binary collision and the one before. The difference is negative because the kinetic energy has decreased due to the collision. Using energy conservation leads to an expression for the impact relative 
velocity

$$
\begin{aligned}
E_{\infty} & =E_{c} \\
\frac{1}{4} m \boldsymbol{v}_{n \infty}^{2} & =\frac{1}{4} m \boldsymbol{v}_{c}^{2}+\Phi(2 a) \\
v_{c}^{2} & = \begin{cases}v_{n \infty}^{2}-v_{n, b}^{2} & \text { for repulsion : } \Phi(2 a)>0 \\
v_{n \infty}^{2}+v_{n, e}^{2} & \text { for attraction : } \Phi(2 a)<0,\end{cases}
\end{aligned}
$$

where Eq. (2.8) has been used in the last line. Using the coefficient of normal restitution for the impact velocity, ignoring the sign, $v_{c}^{\prime}=e v_{c}$, one obtains with Eq. (2.13)

$$
\begin{aligned}
\left(b^{+}-1\right) E_{c} & =\frac{1}{4} m\left(v_{c}^{\prime 2}-v_{c}^{2}\right) \\
& =-\frac{1}{4} m\left(1-e^{2}\right) v_{c}^{2} \\
& \stackrel{(\mathrm{B} 7)}{=}-\frac{1}{4} m\left(1-e^{2}\right) \times \begin{cases}\left(v_{n \infty}^{2}-v_{n, b}^{2}\right) & \text { for repulsion }: \Phi(2 a)>0 \\
\left(v_{n \infty}^{2}+v_{n, e}^{2}\right) & \text { for attraction }: \Phi(2 a)<0 .\end{cases}
\end{aligned}
$$

As compared to the force-free situation, Eq. (B8) shows a decrease of the loss of kinetic energy in the repulsive case, whereas it shows an increase of the loss of kinetic energy in the attractive case. As discussed in the main text, the latter situation is different for separating particles, where the "kinetic energy at infinite-distance"-argument is not plausible.

\section{Acknowledgements}

We want to thank Dietrich Wolf, Olaf Herbst, and Sebastian Gonzalez-Briones for fruitful discussions. Furthermore, we want to acknowledge the financial support of the Deutsche Forschungsgemeinschaft (DFG), of the TUDelft, of the Delft Centre for Computational Science and Engineering (DCSE) and of the faculty of Engineering Technology and the research institute IMPACT of the University of Twente.

\section{References}

[1] M. P. Allen, D. J. Tildesley. Computer Simulation of Liquids. Oxford University Press, Oxford, 1987.

[2] J. C. Almekinders, C. Jones. Multiple jet electrohydrodynamic spraying and applications. J. Aerosol Sci., 30 (1999), 969-971.

[3] J. Barnes, P. Hut. A hierarchical $O(N \log N)$ force calculation algorithm. Nature, 324 (1986), 446-449. 
[4] D. L. Blair, A. Kudrolli. Magnetized granular materials. In H. Hinrichsen and D. Wolf, editors, The Physics of Granular Media., pages 281-296, Weinheim, 2004. Wiley-VCH.

[5] J. Blum, S. Bruns, D. Rademacher, A. Voss, B. Willenberg, M. Krause. Measurement of the translational and rotational Brownian motion of individual particles in a rarefied gas. Phys. Rev. Lett., 97 (2006), 230601.

[6] J. Blum, G. Wurm, S. Kempf, T. Poppe, H. Klahr, T. Kozasa, M. Rott, T. Henning, J. Dorschner, R. Schräpler, H.U. Keller, W.J. Markiewicz, I. Mann, B.A.S. Gustafson, F. Giovane, D. Neuhaus, H. Fechtig, E. Grün, B. Feuerbacher, H. Kochan, L. Ratke, A. El Goresy, G. Morfill, S.J. Weidenschilling, G. Schwehm, K. Metzler, W.-H. Ip. Growth and form of planetary seedlings: Results from a microgravity aggregation experiment. Phys. Rev. Lett., 85 (2000), 2426-2429.

[7] P. Bode, J. P. Ostriker. Tree particle-mesh: An adaptive, efficient, and parallel code of collisionless cosmological simulation. Astrophys. J. Supplem. Series, 145 (2003), No. 1, 1-13.

[8] A. Brahic. Systems of colliding bodies in a gravitational field: I - numerical simulation of the standard model. Astronomy and Astrophysics, 54 (1977), 895-907.

[9] F. G. Bridges, A. Hatzes, D. N. C. Lin. Structure, stability and evolution of Saturn's rings. Nature, 309 (1984), 333-335.

[10] N. V. Brilliantov, T. Pöschel. Deviation from maxwell distribution in granular gases with constant restitution coefficient. Phys. Rev. E, 61 (2000), 2809-2814.

[11] N. V. Brilliantov, T. Pöschel. Kinetic Theory of Granular Gases. Oxford University Press, Oxford, 2004.

[12] N. V. Brilliantov, C. Saluena, T. Schwager, T. Pöschel. Transient structures in a granular gas. Phys. Rev. Let., 93 (2004), No. 13, 134301.

[13] N. V. Brilliantov, F. Spahn, J.-M. Hertzsch, T. Pöschel. A model for collisions in granular gases. Phys. Rev. E, 53 (1996), 5382-5392.

[14] N. F. Carnahan, K. E. Starling. Equation of state for nonattractive rigid spheres. J. Chem. Phys., 51 (1969), No. 2, 635-636.

[15] J. A. Cross. Electrostatics: Principles, Problems and Applications. Adam Hilger, Bristol, 1987.

[16] S. M. Dammer, J. Werth, H. Hinrichsen. Electrostatically charged granular matter. In H. Hinrichsen D. Wolf, editors, The Physics of Granular Media., pages 255-280, WileyVCH, Weinheim, 2004.

[17] S. M. Dammer, D. E. Wolf. Self-focusing dynamics in monopolarly charged suspensions. Phys. Rev. E, 93 (2004), No. 15, 150602. 
[18] J. Du. Hydrostatic equilibrium and Tsallis' equilibrium for self-gravitating systems. Central European Journal of Physics, 3 (2005), No. 3, 376-381.

[19] J. Duran. Sands, Powders and Grains. Springer-Verlag, New York, 2000.

[20] J. W. Eastwood, R. W. Hockney, D. Lawrence. P3M3DP - the 3-dimensional periodic particle-particle-particle-mesh program. Comp. Phys. Commun., 19 (1980), 215-261.

[21] M. H. Ernst. Nonlinear model-Boltzmann equations and exact solutions. Physics Reports, 78 (1981), No. 1, 1-171.

[22] M. H. Ernst, J. R. Dorfman, W. R. Hoegy, J. M. J. van Leeuwen. Hard-sphere dynamics and binary-collision operators. Physica, 45 (1969), No. 1, 127-146.

[23] M. H. Ernst, E. Trizac, A. Barrat. The Boltzmann equation for driven systems of inelastic soft spheres. J. Stat. Phys., 124 (2006), No. 2-4, 549-586.

[24] P. Eshuis, K. van der Weele, D. van der Meer, D. Lohse. The granular Leidenfrost effect: Experiment and theory of floating particle clusters. Phys. Rev. E, 95 (2005), 258001.

[25] S. E. Esipov, T. Pöschel. The granular phase diagram. J. Stat. Phys., 86 (1997), No. 5/6, 1385-1395.

[26] L. W. Esposito, J. N. Cuzzi, J. B. Holberg, E. A. Marouf, G. L. Tyler, C. C. Porco. Saturn's rings, structure, dynamics and particle properties. In Saturn, pages 463-545, Tucson, AZ, Univ. of Arizona Press, 1984.

[27] P. P. Ewald. The calculation of optical and electrostatic grid potential. Ann. d. Physik, 64 (1921), 253-287.

[28] K. B. Geerse. Application of Electrospray: from people to plants. Ph.D. thesis, Technische Universiteit Delft, 2003.

[29] I. Goldhirsch, G. Zanetti. Clustering instability in dissipative gases. Phys. Rev. Lett., 70 (1993), No. 11, 1619-1622.

[30] P. Goldreich. The dynamics of planetary rings. Ann. Rev. Astron. Astrophys., 20 (1982), 249-283.

[31] R. Greenberg, A. Brahic. Planetary Rings. Arizona University Press, Tucson, AZ, 1984.

[32] L. Greengard, V. Rokhlin. A fast algorithm for particle simulations. J. of Comp. Phys., 73 (1987), 325-348.

[33] P. K. Haff. Grain flow as a fluid-mechanical phenomenon. J. Fluid Mech., 134 (1983), 401-430. 
[34] J.-P. Hansen, I. R. McDonald. Theory of Simple Liquids. Academic Press Ltd., London, San Diego, 1990.

[35] D. Henderson. A simple equation of state for hard discs. Molec. Phys., 30 (1975), No. 3, 971-972.

[36] O. Herbst, R. Cafiero, A. Zippelius, H. J. Herrmann, S. Luding. A driven two-dimensional granular gas with coulomb friction. Phys. of Fluids, 17 (2005), No. 10, 107102.

[37] O. Herbst, P. Müller, A. Zippelius. Local heat flux and energy loss in a two-dimensional vibrated granular gas. Phys. Rev. E, 72 (2005) No. 4, 041303.

[38] L. Hernquist. Hierarchical N-body methods. Comp. Phys. Commun., 48 (1988), 107-115.

[39] R. Hoffmann. Modeling and Simulation of an Electrostatic Image Transfer. (Ph.D. thesis) Shaker Verlag, Aachen, 2004.

[40] J. S. Høye. Dynamical pair correlations of classical and quantum fluids perturbed with longrange forces. Physica A, 389 (2010), 1380-1390.

[41] M. Huthmann, A. Zippelius. Dynamics of inelastically colliding rough spheres: Relaxation of translational and rotational energy. Phys. Rev. E, 56 (1997), No. 6, R6275-R6278.

[42] W. Kleber, A. Lang. Triboelectrically charged powder coatings generated by running through holes and slits. J. of Electrostatics, 40\&41 (1997), 237-240.

[43] M. Krause, J. Blum. Growth and form of planetary seedlings: Results from a sounding rocket microgravity aggregation experiment. Phys. Rev. Lett., 93 (2004), 021103.

[44] C. W. J. Lemmens. An Investigation, Implementation and Comparison of 3 important Particle Simulation Techniques: PP: Particle-Particle PM: Particle-Mesh TC: Tree-Code. Report 9746, Faculty of Technical Mathematics and Informatics, Delft, 1997.

[45] M. Linsenbühler, J. H. Werth, S. M. Dammer, H. A. Knudsen, H. Hinrichsen, K.-E. Wirth, D. E. Wolf. Cluster size distribution of charged nanopowders in suspensions. Powder Technology, 167 (2006), No. 3, 124-133.

[46] D. Lohse, R. Bergmann, R. Mikkelsen, C. Zeilstra, D. van der Meer, M. Versluis, K. van der Weele, M. van der Hoef, H. Kuipers. Impact on soft sand: Void collapse and jet formation. Phys. Rev. Let., 93 (2004), No. 19, 198003.

[47] J. Lowell, A. C. Rose-Innes. Contact electrification. Adv. in Phys., 29 (1980), No. 6, 9471023.

[48] S. Luding. Clustering instabilities, arching, and anomalous interaction probabilities as examples for cooperative phenomena in dry granular media. T.A.S.K. Quarterly, Scientific Bulletin of Academic Computer Centre of the Technical University of Gdansk., 2 (1998), No. 3, 417-443. 
[49] S. Luding. Collisions \& contacts between two particles. In H. J. Herrmann, J.-P. Hovi, S. Luding, editors, Physics of dry granular media - NATO ASI Series E350, page 285, Dordrecht, 1998. Kluwer Academic Publishers.

[50] S. Luding. Structure and cluster formation in granular media. Pranama-J. Phys., 64 (2005), No. 6, 893-902.

[51] S. Luding. Cohesive frictional powders: Contact models for tension. Granular Matter, 10 (2008), No. 4, 235-246.

[52] S. Luding. Towards dense, realistic granular media in 2d. Nonlinearity, 22 (2009), R101R146.

[53] S. Luding, A. Goldshtein. Collisional cooling with multi-particle interactions. Granular Matter, 5 (2003), No. 3, 159-163.

[54] S. Luding, H. J. Herrmann. Cluster growth in freely cooling granular media. Chaos, 9 (1999), No. 3, 673-681.

[55] S. Luding, M. Huthmann, S. McNamara, A. Zippelius. Homogeneous cooling of rough, dissipative particles: Theory and simulations. Phys. Rev. E, 58 (1998), 3416-3425.

[56] S. Luding, S. McNamara. How to handle the inelastic collapse of a dissipative hard-sphere gas with the TC model. Granular Matter, 1 (1998), No. 3, 113-128.

[57] S. McNamara. Hydrodynamic modes of a uniform granular medium. Phys. of Fluids A, 5 (1993), 3056-3070.

[58] S. McNamara W. R. Young. Dynamics of a freely evolving, two-dimensional granular medium. Phys. Rev. E, 53 (1996), 5089-5100.

[59] S. Miller. Clusterbildung in Granularen Gasen. (in German). Ph.D. thesis, Universität Stuttgart, 2003.

[60] S. Miller, S. Luding. Cluster growth in two- and three-dimensional granular gases. Phys. Rev. E, 69 (2004), No. 3, 031305.

[61] J. M. Montanero, V. Garzò, M. Alam, S. Luding. Rheology of $2 d$ and $3 d$ granular mixtures under uniform shear flow: Enskog kinetic theory versus molecular dynamics simulations. Granular Matter, 8 (2006), No. 2, 103-115.

[62] M.-K. Müller. Untersuchung von Akkretionsscheiben mit Hilfe der Molekulardynamik. (in German). Diploma thesis, Universität Stuttgart, 2001.

[63] M.-K. Müller. Long-Range Interactions In Dilute Granular Systems. Ph.D. thesis, Universiteit Twente/Enschede, 2007. 
[64] M.-K. Müller, S. Luding. Long-range interactions in ring-shaped particle aggregates. In R. García-Rojo, H.J. Herrmann, S. McNamara, editors, Powders \& Grains, pages 1119-1122, Balkema, Leiden, 2005.

[65] M.-K. Müller, S. Luding. Homogeneous cooling with repulsive and attractive long-range interactions. In M. Nakagawa S. Luding, editors, Powders \& Grains, pages 697-700, AIP Conf. Procs. \#1145, 2009.

[66] M.-K. Müller, T. Winkels, K.B. Geerse, J.C.M. Marijnissen, A. Schmidt-Ott, S. Luding. Experiment and simulation of charged particle sprays. In Proceedings PARTEC 2004, Nuremberg, 2004.

[67] B. Muth, M.-K. Müller, P. Eberhard, S. Luding. Contacts between many bodies. In W. Kurnik, editor, Machine Dynamics Problems, pages 101-114, Warsaw, 2004.

[68] E. Németh. Triboelektrische Aufladung von Kunststoffen. (in German). Ph.D. thesis, Technische Universität Bergakademie Freiberg, 2003.

[69] F. Niermöller. Ladungsverteilung in Mineralgemischen und elektrostatische Sortierung nach Triboaufladung. (in German). Ph.D. thesis, Technische Universität Clausthal, 1988.

[70] J. S. Olafsen, J. S. Urbach. Clustering, order, and collapse in a driven granular monolayer. Phys. Rev. Lett., 81 (1998), 4369.

[71] J. A. G. Orza, R. Brito, T. P. C. van Noije, M. H. Ernst. Patterns and long range correlations in idealized granular flows. Int. J. of Mod. Phys. C, 8 (1997), No. 4, 953-965.

[72] T. Pöschel S. Luding, editors. Granular Gases, Lecture Notes in Physics 564. Springer, Berlin, 2001.

[73] R. Ramírez, T. Pöschel, N. V. Brilliantov, T. Schwager. Coefficient of restitution of colliding viscoelastic spheres. Phys. Rev. E, 60 (1999), 4465-4472.

[74] F. H. Ree, W. G. Hoover. Fifth and sixth virial coefficients for hard spheres and hard disks. J. Chem. Phys., 40 (1964), No. 4, 939-950.

[75] T. N. Scheffler. Kollisionskühlung in elektrisch geladener granularer Materie. (in German). Ph.D. thesis, Gerhard-Mercator-Universität Duisburg, 2000.

[76] T. N. Scheffler, D. E. Wolf. Collision rates in charged granular gases. Granular Matter, 4 (2002), No. 3, 103-113.

[77] T. Schwager, T. Pëoschel. Coefficient of restitution of viscous particles and cooling rate of granular gases. Phys. Rev. E, 57 (1998), 650-654.

[78] F. Spahn, J. Schmidt. Saturn’s bared mini-moons. Nature, 440 (2006), 614-615. 
[79] V. Springel, S. D. M. White, A. Jenkins, C. S. Frenk, N. Yoshida, L. Gao, J. Navarro, R. Thacker, D. Croton, J. Helly, J. A. Peacock, S. Cole, P. Thomas, H. Couchman, A. Evrard, J. Colberg, F. Pearce. Simulating the joint evolution of quasars, galaxies and their large-scale distribution. Nature, 435 (2005), 629-636.

[80] U. Trottenberg, C. W. Oosterlee, A. Schüller. Multigrid. Academic Press, San Diego, 2001.

[81] T. P. C. van Noije, M. H. Ernst, R. Brito. Ring kinetic theory for an idealized granular gas. Physica A, 251 (1998), 266-283.

[82] J. H. Werth, S. M. Dammer, Z. Farkas, H. Hinrichsen, D. E. Wolf. Agglomeration in charged suspensions. Computer Physics Communications, 147 (2002), 259-262.

[83] J. H. Werth, H. Knudsen, H. Hinrichsen. Agglomeration of oppositely charged particles in nonpolar liquids. Phys. Rev. E, 73 (2006), 021402.

[84] J. H. Werth, M. Linsenbuhler, S. M. Dammer, Z. Farkas, H. Hinrichsen, K.-E. Wirth, D. E. Wolf. Agglomeration of charged nanopowders in suspensions. Powder Technology, 133 (2003), 106-112.

[85] D. E. Wolf, T. N. Scheffler, J. Schäfer. Granular flow, collisional cooling and charged grains. Physica A, 274 (1999), 171-181. 